\title{
Zur Homotopietheorie gefaserter Räume
}

\section{Doctoral Thesis}

Author(s):

Eckmann, Beno

Publication date:

1941

Permanent link:

https://doi.org/10.3929/ethz-a-000091765

Rights / license:

In Copyright - Non-Commercial Use Permitted 


\title{
Zur Homotopietheorie gefaserter Räume
}

\author{
Von der
}

Eldgenössischen Technischen Hochschule in Zürich

zur Erlangung der Würde eines Doktors der Mathematik

\author{
genehmigte \\ Promotionsarbeit \\ vorgelegt von \\ Beno Eckmann \\ aus Bern \\ Referent: Herr Prof. Dr. H. Hopf \\ Korreferent: Herr Prof. Dr. F. Gonseth
}

1941

ART. INSTITUT ORELLFOSSLI A.-G., ZORICH 
Auszug aus Commentarii Mathematici Helvetici

vol. XIV, fase. 2 


\title{
Zur Homotopietheorie gefaserter Räume
}

\author{
Von Beno Eckmann, ZüRICH
}

\section{Einleitung}

a) In dieser Arbeit werden topologische Eigenschaften gefaserter Räume mit Hilfe von stetigen Abbildungen untersucht, insbesondere unter Heranziehung der von Hurewicz eingeführten Homotopiegruppen (vgl. Anm. ${ }^{25}$ ). Die Anwendungen der dabei gewonnenen Sätze beziehen sich auf Gruppenräume, Räume von Linienelementen, Felder von Vektoren und Flächenelementen auf Sphären, sowie auf Abbildungen von Sphären auf Sphären niedrigerer Dimension.

b) Unter einer Faserung eines Kompaktums $R$ (nur mit solchen Räumen befassen wir uns) versteht man eine stetige Zerlegung in abgeschlossene Teilmengen, die alle einem Kompaktum $F$ homöomorph sind und die man Fasern nennt; dabei sollen alle zu einer genügend kleinen Umgebung $U$ eines Punktes des Zerlegungsraumes $Z$ (der auch Faserraum genannt wird) gehörigen Fasern in $R$ das topologische Produkt $F \times U$ bilden. Die stetige Abbildung von $R$ auf $Z$, die jedem Punkt von $R$ die Faser zuordnet, auf welcher er liegt, heißt Projektion oder Faserabbildung $P$.

Beispiele von Räumen, die in natürlicher Weise gefasert sind, bilden - abgesehen vom trivialen Fall der topologischen Produkte - die Gruppenräume (in der Zerlegung in Restklassen nach einer abgeschlossenen Untergruppe) und die Räume der Linienelemente (Flächenelemente, Vektorsysteme usw.) differenzierbarer Mannigfaltigkeiten; man kennt außerdem besonders einfache Faserungen von Sphären (vgl. Nr. 2). Eine allgemeine Theorie der gefaserten Räume, die möglichst wenige Voraussetzungen benützt, wird also ein großes und vielseitiges Anwendungsgebiet haben, und ihre Aussagen lassen sich verfeinern, sobald man sich auf speziellere Fälle beschränkt. Faserungen sind bisher insbesondere von Seifert, Feldbau, Gysin (vgl. ${ }^{9}$ ) und Whitney ${ }^{6}$ ) untersucht worden.*)

c) Unser Beitrag zur Untersuchung gefaserter Räume bezieht sich sowohl in der Fragestellung als auch in der Methode auf Homotopieeigenschaften. Alle unsere Aussagen beruhen auf einer Voraussetzung,

*) Zusatz bei der Korrektur: In einer C. R.-Note von C. Ehresmann und J. Feldbau (Sur les propriétés d'homotopie des espaces fibrés, C. R. Paris 211 (1941) 945-948), die mir soeben zugegangen ist, werden ohne Beweis Sätze über Faserungen angekün. digt, die mit gewissen Teilresultaten der vorliegenden Arbeit zusammenfallen, nämlich mit dem „Lemma" (Nr. 3d) und den „Hurewicz'schen Formeln" (Nr. 6). 
die uns für die Untersuchung stetiger Abbildungen und ihrer Deformationen angemessen und bequem erscheint: daß es sich um Zerlegungen handelt, die in einem im folgenden (Nr. 1c) präzisierten Sinne „retrahierbar" sind. Für hinreichend reguläre Faserungen ist diese Voraussetzung immer erfüllt; anderseits werden andere Eigenschaften von Faserungen, also auch die eingangs als Definition angeführten, nie benützt. Wir sprechen deshalb in unseren Ausführungen nie von Faserungen, sondern formulieren alle Aussagen für retrahierbare Zerlegungen, oder, da viele Sätze einen noch weitern Gültigkeitsbereich haben, für retrahierbare Überdeckungen. - In dieser Einleitung soll allerdings nur von (regulären) Faserungen die Rede sein.

d) Ist $f$ eine Abbildung eines Kompaktums $X$ in $R$, so heißt die Abbildung $P f$ von $X$ in $Z$ die Spur von $f$ (vgl. Nr. 1d, lf). Der Kern unserer Betrachtungen ist ein Lemma (Nr. 3d), das im wesentlichen aussagt, daß jede zu einer Spur homotope Abbildung selbst eine Spur ist. Die Abbildungssätze (Nr. 4), sowie die Zusammenhänge zwischen den Homotopiegruppen von $R, F$ und $Z$ (Nr. 6, 7), die sich daraus ergeben, stellen eine weitgehende Verallgemeinerung von Sätzen dar, die Hurewicz (a. a. O.8) für Restklassenzerlegungen von Gruppenräumen angegeben hat. Von unsern Abbildungssätzen heben wir hier die folgenden hervor:

1. Wenn $R$ auf sich wesentlich ist, d. h. wenn die identische Abbildung von $R$ auf sich eine wesentliche Abbildung ist, so ist die Faserabbildung $P$ eine wesentliche Abbildung von $R$ auf $Z$ (Nr. 5, Satz 1). Für diesen Satz ist unseres Wissens in dieser Allgemeinheit auch mit algebraischen Hilfsmitteln kein anderer Beweis bekannt. (Für Faserungen geschlossener Mannigfaltigkeiten in Sphären ist der Satz von Gysin a. a. 0. ${ }^{9}$ ) mit Homologiemethoden bewiesen worden). Er ist im folgenden Satz enthalten:

2. Die Spur einer wesentlichen Abbildung ist wesentlich (Nr. 4, Satz C).

Wichtig ist ferner (man vergleiche den Beweis eines Satzes von Wazewski in Nr. 4d):

3. Eine Abbildung in $Z$, die sich auf einen Punkt zusammenziehen läßt, ist eine Spur (Nr. 4, Satz A).

e) Unter einer Schnittfläche einer Faserung verstehen wir eine Abbildung von $Z$ in $R$, deren Spur die Identität von $Z$ ist (also eine topologische Abbildung von $Z$ in $R$, bei welcher das Bild jede Faser genau einmal trifft). Im Falle der Linienelementräume bedeutet eine Schnittfläche ein Feld (damit ist immer ein stetiges singularitätenfreies Feld gemeint) von Linienelementen, im Falle einer Gruppenzerlegung das Zerfallen des Gruppenraumes in ein topologisches Produkt (vgl. Nr. 11). 
Die Frage, ob eine Faserung eine Schnittfäche besitzt, wird in einem besondern Abschnitt ( $\$ 4)$ behandelt; notwendig für die Existenz einer Schnittfläche ist unter anderem, daß die Homotopiegruppen von $R$ dieselbe Struktur haben wie beim topologischen Produkt von $F$ und $Z$ (Nr. 9, Satz G, II). Wenn der Zerlegungsraum $Z$ eine Sphäre ist, können wir notwendige und hinreichende Bedingungen für die Existenz einer Schnittfläche angeben, und auch für den Fall der Nicht-Existenz die Struktur der Faserung, insbesondere die Homotopiegruppen, näher untersuchen (Schnittelemente, Nr. 10).

f) Von den Resultaten, die wir durch Anwendung der allgemeinen Sätze auf spezielle Faserungen erhalten, seien hier einige erwähnt:

1. Mit Hilfe der bekannten Sphärenfaserungen (Nr. 2f) können wir die Existenz wesentlicher Abbildungen der Sphäre $S^{N}$ auf die Sphäre $S^{n}$ in einigen neuen, allerdings sehr speziellen Fällen nachweisen, so für $N=8$ oder 10 und $n=4$, für $N=16$ oder 18 oder 22 und $n=8$. Man kann nämlich (Nr. 5, Satz $3^{\prime}$ ) jedes Kompaktum, das sich wesentlich auf $S^{7}$ abbilden läßt, auch wesentlich auf $S^{4}$ und jedes Kompaktum, das sich wesentlich ouf $S^{15}$ abbilden läßt, wesentlich ouf $S^{8}$ abbilden.

2. Für die Homotopiegruppen der orthogonalen und der unitär-unimodularen Gruppen gelten Aussagen allgemeiner Natur: die k-te Homotopiegruppe hat für, ,fast alle" orthogonalen bzw. für ,fast alle“ unitärunimodularen Gruppen dieselbe Struktur (Nr. 8, Satz 9 und 10).

3. Zurückführung der Homotopiegruppen der komplexen und quaternionalen projektiven Räume auf diejenigen der Sphären (Nr. 8a).

4. Bestimmung einiger Homotopiegruppen der Linienelementräume der Sphären.

5. Wir zeigen, daß es auf der Sphäre $S^{5}$ kein 2-Feld gibt, d. h. kein System von 2 tangentialen Vektorfeldern, die in jedem Punkt der $S^{5}$ linear unabhängig sind.

Zum Beweis dieses Satzes (Nr. 16) benützen wir außer unsern Sätzen (eine wichtige Rolle spielt dabei die Kenntnis von Homotopiegruppen des Linienelementraumes der $S^{4}$ ) einen Satz von Pontrjagin (vgl. ${ }^{52}$ ) über unitäre Gruppen.

Eine Mannigfaltigkeit der Dimension $n$ heißt parallelisierbar, wenn es auf ihr ein System von $n$ linear unabhängigen tangentialen Vektorfeldern gibt. Aus 5. folgt, daß die Sphäre $S^{5}$ nicht parallelisierbar ist. Damit ist eine Frage beantwortet, die zwar spezieller Natur ist, aber doch einiges Interesse verdient, und die sich besonders im Anschluß an Arbeiten von Stiefel (vgl. ${ }^{14}$ ) und ${ }^{53}$ ) aufdrängt: Man weiß, daß die Sphären $S^{1}, S^{3}$ 
und $S^{7}$ parallelisierbar sind, und daß es auf Sphären gerader Dimension nicht einmal ein tangentiales Vektorfeld gibt. Für alle andern Dimensionszahlen $n$ war die Frage, ob die Sphäre $S^{n}$ parallelisierbar ist, bisher noch offen; durch unser Resultat wird sie wenigstens für $n=5$ entschieden. *)

6. Auf Sphären gerader Dimension $n \geqslant 4$ und auf der Sphäre $S^{5}$ gibt es kein Feld von tangentialen 2-dimensionalen Flächenelementen.

Dieser Satz ergibt sich daraus, daß jedes Feld von tangentialen Flächenelementen auf einer Sphäre ,,durch ein 2-Feld aufgespannt“ werden kann (Nr. 17, Satz 29).

g) Diese Resultate sind z.T., besonders hinsichtlich der darin auftretenden Dimensionszahlen, sehr speziell. Es sei erwähnt, daß der Versuch, sie in naheliegender Weise auszudehnen, oft daran scheitert, daß die Homotopiegruppen der Sphären und anderer Räume noch in vielen Fällen unbekannt sind, wie denn überhaupt eine grundsätzliche Methode zur Bestimmung der Homotopiegruppen noch nicht existiert. Wegen dieses letztern Umstandes möchten wir auch darauf hinweisen, daß manchmal Faserungen als Hilfsmittel zur Bestimmung von Homotopiegruppen herangezogen werden können, wobei die in Nr. 6 und 7 aufgestellten Beziehungen, die wir die Hurewicz'schen Formeln nennen, eine besondere Rolle spielen; diese "Methode der Faserungen" hat bei uns in einigen Fällen zum Ziel geführt, wird aber natürlich im allgemeinen nicht ausreichen.

\section{\$ 1. Retrahierbare Uberdeckungen und Zerlegungen}

\section{Definitionen}

a) Die Gesamtheit der abgeschlossenen Punktmengen eines Kompaktums $R$ wird selbst zu einem kompakten metrischen $\left.\operatorname{Raum}^{1}\right) \mathfrak{A}(R)$, wenn man als Entfernung $\varrho(A, B)$ zweier Punkte $A, B \in \mathfrak{A}(R)$ die ,Abweichung“" der zugehörigen Punktmengen $\bar{A}, \bar{B} \subset R$ einführt; darunter versteht $\operatorname{man}^{1}$ ) die untere Grenze der den Bedingungen ${ }^{2}$ )

*) Zusatz bei der Korrektur : Ich habe inzwischen beweisen können, daß es allgemeiner auf allen Sphären der Dimensionen $4 k+1$ kein 2-Feld gibt; der Beweis, in dem übrigens eine Heranziehung unitärer Gruppen nicht mehr nötig ist, wird demnächst erscheinen.

1) Alexandroff-Hopf, Topologie (Berlin 1935); S. 112 und 115. - Bezüglich aller auftretenden Begriffe der mengentheoretischen Topologie verweisen wir auf den ersten Teil dieses Buches.

2) Für eine Punktmenge $M \subset R$ bezeichnen wir mit $U(M, \varepsilon)$ die $\varepsilon$-Umgebung von $M$, d. h. die Menge aller Punkte $p$ mit $\rho(M, p)=\inf _{q \in M} \rho(q, p)<\varepsilon$ (wo $\rho(x, y)$ die Entfernung von $x$ und $y \in R$ bedeutet). 


$$
\bar{A} \subset U(\bar{B}, \alpha), \quad \widetilde{B} \subset U(\bar{A}, \alpha)
$$

genügenden Zahlen $\alpha$. Aus $\varrho(A, B)<\varepsilon$ folgt also

$$
\bar{A} \subset U(\bar{B}, \varepsilon) \text { und } \bar{B} \subset U(\bar{A}, \varepsilon) \text {. }
$$

Wir halten immer an folgender Bezeichnungsweise fest: Für jeden Punkt $A$ von $\mathfrak{A}(R)$ bedeutet $\bar{A}$ die betreffende Punktmenge von $R$ ( $\bar{A}$ und $A$ bedeuten also dasselbe, einmal als Punktmenge von $R$, einmal als Punkt von $\mathfrak{A}(R)$ aufgefaßt).

b) Eine abgeschlossene Teilmenge $Z \subset \mathfrak{A}(R)$ induziert eine Überdeckung 3 von $R$ mit abgeschlossenen Mengen, wenn zu jedem $a \in R$ ein $A \in Z$ existiert mit $a \in \bar{A}$. Die den Punkten $A \in Z$ entsprechenden Mengen $\bar{A} \subset R$ sind die Elemente der Überdeckung 3 , und $Z$ soll Überdeckungsraum von 3 heißen; $Z$ ist ein Kompaktum. Wir betrachten nur Überdeckungen, deren Elemente echte Teilmengen von $R$ sind. Bei einer topologischen Abbildung des Kompaktums $R$ auf ein Kompaktum $R_{1}$ geht die Überdeckung 3 von $R$ in naheliegender Weise über in eine Überdeckung $Z_{1}$ von $R_{1}$. Zwei Überdeckungen 3 von $R$ und $3_{1}$ von $R_{1}$ heißen homöomorph, wenn es eine topologische Abbildung von $R$ auf $R_{1}$ gibt, bei welcher 3 in $\mathfrak{Z}_{1}$ übergeht.

c) Definition: Die Überdeckung 3 von $R$, mit dem Überdeckungsraum $Z$, heißt retrahierbar, wenn es eine Zahl $r>0$ und zu jedem Punkt $A \in Z$ eine stetige Abbildung $Q$ von $U(\bar{A}, r)$ auf $\bar{A} \subset R$ gibt, die auf $\bar{A}$ selbst die Identität ist, und die auch von $A \in Z$ stetig abhängt. Es soll also $Q(A, b)$ definiert und stetig sein für $A \in Z, b \in U(\bar{A}, r)$, und es soll gelten

und

$$
Q(A, b)=a \epsilon \bar{A}
$$

$$
Q(A, b)=b, \text { falls } b \in \bar{A} .
$$

Daß die Überdeckung 3 von $R$ retrahierbar ist, besagt also: Jedes Element $\bar{A}$ besitzt eine Umgebung in $R$, die sich auf $\bar{A}$ retrahieren läßt, und zwar so ,daß die Retraktion mit $\bar{A}$ stetig variiert; dabei genügt die Größe dieser Umgebungen einer Gleichmäßigkeitsbedingung ( $r$ von $A$ unabhängig). Die Forderung der Retrahierbarkeit schränkt natürlich den Bereich der zugelassenen Utberdeckungen wesentlich ein $^{3}$ ).

$\left.{ }^{3}\right)$ So folgt z. B. aus der Retrahierbarkeit einer Überdeckung 3 , daß die Elemente $\bar{A}$ ,den gleichen Homotopietypus" haben (wegen dieses Begriffes vgl. man W. Hureuricz, Beiträgezur Topologieder Deformationen III (Proc. Akad. Amsterdam, 39 [1936], 112-126, insbes. S. 125); ebenso, daß sie gleiche Dimension haben. 
Die in der Definition genannte Abbildung $Q(A, b)$ ist bei festem $B \in Z$ und variablem $A \in U(B, r)$ für jedes $b \in \bar{B}$ eine stetige Abbildung von $U(B, r) \subset Z$ in $R$ mit der ,Anfangsbedingung “ $Q(B, \mathrm{~b})=b$, die auch von $b$ stetig abhängt (in der Tat: wenn $A \in U(B, r)$, so ist $b \in U(\bar{A}, r)$, also $Q(A, \mathrm{~b})$ definiert und stetig). Diese Abbildung ist gleichmäßig stetig in folgendem Sinne:

$\mathrm{Zu}$ jedem $\varepsilon>0$ gibt es eine von $A, A^{\prime} \in Z$ und $b \in R$ unabhängige Zahl $\delta>0$, so $\operatorname{da} \varrho\left(Q(A, b), Q\left(A^{\prime}, b\right)\right)<\varepsilon$, wenn $\varrho\left(A, A^{\prime}\right)<\delta$.

Beweis: Es sei $0<r^{\prime}<r$, und $T$ sei die Menge derjenigen Punkte $(A, b)$ des (metrisierten) topologischen Produkts $Z \times R$, für welche $\varrho(\bar{A}, b) \leqslant r^{\prime}<r$ ist; wir zeigen zunächst, da $\beta T$ in $Z \times R$ abgeschlossen ist.

$\left(A_{n}, b_{n}\right)$ sei eine Folge von Punkten aus $T$, die gegen $(A, b) \in Z \times R$ konvergiert; bei beliebigem $h>0$ gilt also für genügend große $n$

$$
\varrho\left(A_{n}, A\right)<h \quad \text { und } \quad \varrho\left(b_{n}, b\right)<h \text {. }
$$

Ferner gibt es wegen $\varrho\left(\bar{A}_{n}, b_{n}\right) \leqslant r^{\prime}$ einen Punkt $a_{n} \in \bar{A}_{n}$, so daß

$$
\varrho\left(a_{n}, b_{n}\right) \leqslant r^{\prime},
$$

und zu $a_{n}$ gibt es einen Punkt $a \in \bar{A}$, so da $B$

ist. Also gilt

$$
\varrho\left(a, a_{n}\right)<h
$$

$$
\varrho(\bar{A}, b) \leqslant \varrho(a, b) \leqslant \varrho\left(a, a_{n}\right)+\varrho\left(a_{n}, b_{n}\right)+\varrho\left(b_{n}, b\right) \leqslant r^{\prime}+2 h,
$$

also, weil $h$ beliebig ist, $\varrho(\bar{A}, b) \leqslant r^{\prime}$, d. h. $(A, b) \in T$, somit ist $T$ abgeschlossen in $Z \times R$.

Daraus folgt, daß $T$ ein Kompaktum ist, und als Abbildung dieses Kompalktums in $R$ ist $Q(A, b)$ gleichmäßig stetig in bezug auf $(A, b) \in T$, also auch in bezug auf $A \in Z, b \in U\left(\bar{A}, r^{\prime}\right)$. Darin ist aber die Behauptung enthalten (wenn man die in der Definition der Retrahierbarkeit auftretende Zahl $r$ durch eine etwas kleinere ersetzt).

Im folgenden soll immer, auch ohne besondere Erwähnung, $R$ ein Kompaktum, 3 eine retrahierbare Überdeckung von $R$ und $Z$ den zugehörigen Überdeckungsraum bezeichnen.

d) Wir bezeichnen mit $Y^{X}$ den ,Abbildungsraum" eines Kompaktums $X$ in einen beschränkt metrisierten Raum $Y$ : die Punkte von $Y^{X}$ sind die stetigen $^{4}$ ) Abbildungen $f$ von $X$ in $Y$, seine Metrik ist durch

4) Alle betrachteten Abbildungen sind stetig; wir lassen dieses Beiwort gewöhnlich weg. 
gegeben.

$$
\varrho(f, g)=\operatorname{Max}_{x \in X} \varrho(f(x), g(x)),
$$

Definition: Wir nennen $F \in Z^{X}$ eine Spur von $f \in R^{X}$ (bezüglich der Uberdeckung 3 von $R$ ),

$$
F=S p f
$$

wenn für jedes $x \in X$ gilt $: f(x) \in \overline{F(x)}$.

Es fragt sich nun, welche Abbildungen $f \in R^{X}$ Spuren besitzen, und welche Abbildungen $F \in Z^{X}$ Spuren sind. Die wichtigste Aussage hierüber wird ein später ( $\$ 2, \mathrm{Nr} .3 \mathrm{~d}$ ) formuliertes Lemma sein; unter spezielleren Voraussetzungen über 3 , die wir im folgenden untersuchen, lassen sich außerdem noch einige, eher triviale, Aussagen machen.

e) Eine Spur der Identität $i$ von $R$ ist eine Abbildung $I \in Z^{R}$, bei welcher für jedes $a \in R$ gilt: $\quad a \in \overline{I(a)}$.

Wenn es in 3 eine Spur der Identität gibt, so besitzt jede Abbildung $f \in R^{X}$ (mindestens) eine Spur $F$, nämlich $F^{\prime}=I f$ :

$$
F(x)=I(f(x)) \text {, also } f(x) \in \overline{F(x)} .
$$

Wir bezeichnen die Bildmenge $I(R) \subset Z$ mit $Z^{\prime}$; wenn speziell (vgl. Nr. 1f) für jedes $A \in Z^{\prime}$ gilt

$$
I Q(A, b)=A,
$$

dann bildet $Q(A, b)$ die Umgebung $U(I b, r) \subset Z^{\prime}$ topologisch in $R$ ab, und $I$ ist eine gebietstreue Abbildung von $R$ auf $Z^{\prime}$.

f) Die Überdeckung 3 von $R$ heißt eine Zerlegung, wenn für alle $a \epsilon R$ aus $a \in \bar{A}$ und $a \epsilon \bar{B}(A, B \in Z)$ folgt: $A=B$. Wir verstehen dann unter der Projektion $P$ diejenige Abbildung von $R$ auf $Z$, die jedem Punkt $a \in R$ die Punktmenge, in der er liegt, d. h. denjenigen Punkt $A \in Z$, für welchen $a \in \bar{A}$ ist, zuordnet; es ist also für alle $A \in Z$

$$
P^{-1} A=\bar{A} \text {. }
$$

Zerlegungen sollen immer stetig $\operatorname{sein}^{5}$ ); das bedeutet: zu jedem $\varepsilon>0$ gibt es ein $\delta>0$, so da $\beta$ aus $\varrho(a, \bar{B})<\delta$ und $a \in \bar{A}$ folgt $\varrho(A, B)<\varepsilon$; dann ist aber die Abbildung $P$ stetig, und $P(R)=Z$ ist der Zerlegungsraum $^{5}$ ) der Zerlegung 3 .

5) Wegen der Terminologie und der einfachsten Eigenschaften der Zerlegungen vgl. $\operatorname{man}{ }^{1}$ ), S. 92 und 98. 
Die Projektion $P$ ist eine Spur der Identität von $R$, und für jedes $A \in Z$ gilt

$$
P Q(A, b)=A
$$

somit ist (vgl. Nr. 1e) $P$ eine gebietstreue Abbildung von $R$ auf $Z$ und $Q(A, b)$ ihre ,lokale eindeutige Umkehrung".

In einer Zerlegung 3 ist eine Abbildung $F \in Z^{X}$ dann und nur dann Spur von $f \in R^{X}$, wenn $F=P f i s t$.

Denn $F(x)=P(f(x))$ ist gleichbedeutend mit $f(x) \epsilon \overline{F(x)} .-P f \in Z^{X}$ soll Projektion der Abbildung $f \in R^{X}$ heißen; in einer Zerlegung sind also diejenigen Abbildungen aus $Z^{X}$, die Spuren sind, identisch mit den Projektionen der Abbildungen aus $R^{x}$.

Wir sagen, die Utberdeckung 3 enthalte eine Zerlegung $3^{\prime}$, wenn der Überdeckungsraum $Z$ eine Teilmenge $Z^{\prime}$ enthält, die eine Zerlegung von $R$ induziert. Dann gilt für die zugehörige Projektion $P$ von $R$ auf $Z^{\prime} \subset Z$ alles soeben Gesagte.

g) Wenn die Identität $J$ von $Z$ eine Spur ist, d. h. wenn es eine Abbildung $j \in R^{Z}$ mit $j(A) \in \bar{A}$ für alle $A \in Z$ gibt, so ist jede Abbildung $F \in Z^{X}$ eine Spur, nämlich Spur von $f=j F$; denn es ist

$$
f(x)=j(F(x)) \in \overline{F(x)} .
$$

Wenn 3 eine Zerlegung ist, und die Identität $J$ von $Z$ eine Spur ist, d. h. wenn es eine Abbildung $j \in R^{Z}$ gibt mit $J=P j$, so nennen wir $j$ eine Schnittfläche der Zerlegung; $j$ ist dann eine topologische Abbildung von $Z$ in $R$, die jedem Punkt $A \in Z$ einen Punkt $a \in \bar{A}$ zuordnet.

Wir werden die Schnittflächen später $(\S 4)$ näher untersuchen.

h) Es sei noch bemerkt, daß man jeder Überdeckung 3 von $R$ eine Zerlegung $3^{*}$ eines Kompaktums $R^{*}$ zuordnen kann: $R^{*}$ sei die Menge derjenigen Punkte $(A, a)$ des topologischen Produktes $Z \times R$, für welche $a \in \bar{A}$ ist; dann bilden die Punkte $(A, a)$ mit festem $A$ eine der Menge $\bar{A} \subset R$ homöomorphe Teilmenge $\tilde{A} \subset R^{*}$, und diese Mengen $\tilde{A}$ bilden eine stetige Zerlegung $3^{*}$ von $R^{*}$, deren Zerlegungsraum $Z^{*}$ dem Überdeckungsraum $Z$ homöomorph ist $\left.{ }^{6}\right)$. $3^{*}$ ist übrigens von selbst retrahierbar, wenn 3 es ist. Die Beziehung zum Raum $R$ kommt darin zum Ausdruck, daß auch $R$ ein Zerlegungsraum von $R^{*}$ ist (Elemente dieser

$\left.{ }^{8}\right)$ In der Theorie der "sphere-spaces" von $H$. Whitney (Bull. of the Am. Math. Soc., 1937, 785-800) entspricht dem Raum $R$ der Basisraum $K$ des Sphärenraumes $S(K)$, dem Raum $R^{*}$ der ,,totàle Raum" $\subseteq(K)$ (wenn der Sphärenraum $S(K)$ durch eine Überdeckung von $K$ mit Sphären gegeben ist). 
Zerlegung sind die Teilmengen von $R^{*}$, die aus den Punkten $(A, a)$ mit festem $a$ bestehen).

In einem gewissen Sinne läßt sich also jede Überdeckung als Zerlegung auffassen, was für die weiteren Untersuchungen bequemer ist. Unsere Anwendungen handeln dementsprechend auch fast ausschließlich von Zerlegungen.

\section{Beispiele}

Die folgenden Beispiele retrahierbarer Zerlegungen und Überdeckungen sollen nicht nur die eingeführten Begriffe illustrieren, sondern auch später zu Anwendungen herangezogen werden.

a) Das topologische. Produkt $X \times Y$ zweier Kompakten $X$ und $Y$ ist in natürlicher Weise zerlegt in die Mengen der Punkte $(x, y)$ mit festem $y \in Y$. Wir nennen diese Zerlegung die "Links-Zerlegung" von $X \times Y$; der zugehörige Zerlegungsraum ist dem Raum $Y$ homöomorph, und die Zerlegung ist offenbar retrahierbar. Sie besitzt eine Schnittfläche:

$$
j(y)=\left(x_{0}, y\right) \in X \times Y
$$

mit beliebigem festem $x_{0} \in X$. - (Analoges gilt für die „Rechts-Zerlegung".)

b) Die (unverzweigte) Überlagerungsmannigfaltigkeit mit endlicher Blätterzahl $k$ einer geschlossenen Mannigfaltigkeit $Z$ läßt sich zerlegen in die Mengen $\bar{A}$ der Punkte $a_{1}, \ldots, a_{k}$, die ,über" einem Punkt $A \in Z$ liegen. Daß diese Zerlegung retrahierbar ist, zeigt folgende Abbildung $Q(A, b): r>0$ sei so gewählt, daß für jedes $A \in Z$ die Umgebung $U(\bar{A}, r)$ aus den $k$ homöomorphen Umgebungen $U\left(a_{i}, r\right)(i=1, \ldots, k)$ besteht; dann setzen wir

$$
Q(A, b)=a_{i} \text { für } b \in U\left(a_{i}, r\right)(i=1, \ldots, k) .
$$

Hingegen ist diese ,natürliche“ Zerlegung einer Überlagerungsmannigfaltigkeit nicht mehr retrahierbar, wenn Verzweigungspunkte auftreten (wie bei Riemann'schen Flächen als Überlagerungen der Kugel).

c) $G$ sei eine kompakte topologische Gruppe, $U$ eine abgeschlossene Untergruppe von $G$. Die Restklassen von $G$ nach $U$ bilden eine Zerlegung 3 von $G$; ihr Zerlegungsraum $Z$ ist ein Wirkungsraum von $G$. (Ein Kompaktum $W$ heißt Wirkungsraum von $G$, wenn es eine transitive Gruppe topologischer Transformationen von $W$ auf sich gibt, die stetig homo- 
morphes Bild von $G$ ist' $)$ ). Umgekehrt gehört zu jedem Wirkungsraum $W$ von $G$ eine Zerlegung von $G$ in Restklassen nach der sog. Isotropiegruppe, d. h. der (abgeschlossenen) Untergruppe derjenigen Transformationen von $W$, die einen bestimmten Punkt von $W$ festlassen, und $W$ ist dem Zerlegungsraum homöomorph (das gilt im allgemeinen nur für kompakte Gruppen).

In seinen Untersuchungen über solche Gruppenzerlegungen setzt Hurewicz ${ }^{8}$ ) eine der Retrahierbarkeit verwandte Eigenschaft voraus, die insbesondere für Lie'sche Gruppen immer erfüllt ist; man kann daraus entnehmen (und übrigens auch leicht direkt einsehen), daß die Restklassenzerlegung einer geschlossenen Lie'schen Gruppe nach einer abgeschlossenen Untergruppe immer retrahierbar ist.

d) Diese Zerlegungen Lie'scher Gruppen sind vom geometrischen Standpunkt aus Beispiele für die sogenannten Faserungen ${ }^{9}$ ): Eine Zerlegung 3 des Kompaktums $R$ heißt Faserung, wenn ihre Elemente, „Fasern" genannt, alle einem Kompaktum $F$ homöomorph sind, und wenn für jede hinreichend kleine Umgebung $U \subset Z$ die Menge $P^{-1} U \subset R$ (also die Vereinigungsmenge $\Sigma \bar{A}$ ) dem topologischen Produkt $F \times U$, $A \in U$

und die durch 3 induzierte Zerlegung von $P^{-1} U$ der Links-Zerlegung von $F \times U$ homöomorph ist; die Projektion $P$ heißt in diesem Fall auch Faserabbildung, der Zerlegungsraum $Z$ Faserraum.

Wenn $R$ und $F$ geschlossene Riemann'sche Mannigfaltigkeiten sind, und die Fasern hinreichend oft differenzierbar in $R$ liegen, so ist die Zerlegung retrahierbar; man kann leicht eine allen Bedingungen der Definition genügende Retraktionsabbildung angeben, bei welcher die Umgebung jeder Faser $F$ längs den zu $F$ orthogonalen geodätischen Linien auf die Faser ,zusammengezogen" wird. Zu diesen ,,regulären Faserungen" gehören auch alle im folgenden untersuchten Zerlegungen; wir werden indessen hievon keinen Gebrauch machen, sondern den Beweis der Retrahierbarkeit durch direkte Angabe besonders einfacher Abbildungen $Q(A, b)$ erbringen.

7) Man vgl. E. Cartan, Groupes finis et continus et l'Analyse Situs (Mémorial des sc. math. 42, Paris 1930), S. 13 (Wirkungsraum heißt dort „espace homogène").

8) Beiträge zur Topologie der Deformationen I (Proc. Amsterdam 38 [1935], 112-119), S. 116 unten.

-) Vgl. J. Feldbau C. R. 208 (1939), S. 1621. Ferner: H. Seifert, Topologie dreidimensionaler gefaserter Räume (Acta math. 60 [1932]); es sei bemerkt, daß die Forderung der Retrahierbarkeit das Auftreten von "Ausnahmefasern" im Sinne von Seifert ausschließt. - W. Gysin hat kürzlich Faserungen geschlossener Mannigfaltigkeiten in Sphären mit Methoden der Homologietheorie untersucht. (Comm. math. helv., Vol. 14.) 
Bei Faserungen der oben beschriebenen Art werden wir sowohl für die Zerlegung 3 selbst als auch für den Zerlegungsraum $Z$ oft die (der Gruppentheorie entnommene) Bezeichnung

$$
R / F
$$

verwenden und durch eine Gleichung der Form

$$
R / F=M
$$

die Homöomorphie des Zerlegungsraumes zu einem Raum $M$ zum Ausdruck bringen.

e) Uberdeckung der Sphären mit Groß-Sphären. Wir betrachten im $n$-dimensionalen euklidischen Raum $R^{n}$ das Büschel aller $q$-dimensionalen Ebenen $E^{q}(0<q<n)$ durch einen festen Punkt $0 \in R^{n}$; diese Ebenen schneiden die um 0 gelegte Einheitssphäre $S^{n-1}$ des $R^{n}$ in $(q-1)$-dimensionalen Groß-Sphären $S^{q-1}$, die eine Überdeckung 3 der $S^{n-1}$ bilden; den zugehörigen Überdeckungsraum $Z$ nennen wir den Großkreiseraum $G_{n-1}, q-1$. Diese Utberdeckung ist retrahierbar; wir konstruieren zum Beweis folgende Abbildung $Q(A, b)$ für $A \in Z, b \in S^{n-1}$ :

$N(A, b)$ sei die Normalprojektion des Punktes $b \in S^{n-1}$ auf die Ebene $E^{q}$, in welcher $\bar{A}$ (das ist eine $S^{q-1}$ ) liegt; bedeutet $|y|$ den Abstand des Punktes $y \in R^{n}$ von 0 , so ist $|N(A, b)|$ eine gleichmäßig stetige Funktion von $A \in Z, b \in S^{n-1}$; da ferner $|N(A, b)|=1$ ist für $b \in \bar{A}$, so gibt es eine Zahl $r>0$, so da $|N(A, b)|>0$ ist, wenn $b \in U(\bar{A}, r)$. Setzen wir nun $\left.{ }^{10}\right)$

$$
Q(A, b)=\frac{1}{|N(A, b)|} \cdot N(A, b),
$$

so ist dies eine stetige Abbildung von $U(\bar{A}, r)$ auf $\bar{A}$, die auch von $A \in Z$ stetig abhängt und auf $\bar{A}$ die Identität ist.

f) Sphärenfaserungen. Die eben beschriebene Überdeckung $3 \mathrm{der} S^{n-1}$ mit Groß-Sphären enthält eine Zerlegung $3^{\prime}$, wenn es möglich ist, im Büschel der Ebenen $E^{q}$ ein schlichtes Büschel auszuzeichnen, d. h. eine Schar von Ebenen $E^{q}$ durch 0 , derart, daß durch jeden von 0 verschiedenen Punkt $y$ des $R^{n}$ genau eine Ebene der Schar geht, die stetig mit $y$ variiert. Solche schlichte Büschel existieren nur für gewisse Dimensionszahlen $n$ und $q^{11}$, jedenfalls für $n=k q$ mit $q=1,2,4$ und $k=$

10) $O$ sei Ursprung eines Koordinatensystems im $R^{n}$, und $\lambda y$ der Punkt, dessen Koordinaten das $\lambda$-fache der Koordinaten von $y \in R^{n}$ sind.

11) Die genauen Bedingungen für $n$ und $q$ sind uns unbekannt; es sei aber bemerkt, daß Gysin a. a. $\left.0 .{ }^{9}\right)$ notwendige Bedingungen angegeben hat: $q$ muß Teiler von $n$, und $q$ muß gerade sein (oder $=1$ ). 
$2,3, \ldots$, außerdem mit $q=8$ und $k=2$; in diesen Fällen kann man sie nämlich nach Hopf ${ }^{12}$ ) unter Heranziehung hyperkomplexer Zahlsysteme $\mathfrak{S}_{q}$ festlegen $\left(\mathfrak{S}_{1}=\right.$ reelle Zahlen, $\mathfrak{S}_{2}=$ komplexe Zahlen, $\mathfrak{S}_{4}=$ Quaternionen, $\mathfrak{S}_{8}=$ Cayley'sche Zahlen ${ }^{12}$ )). Die gemäß e) retrahierbaren ${ }^{13}$ ) Zerlegungen $3^{\prime}$ der $S^{n-1}$ in $(q-1)$-dimensionale Groß-Sphären, die man so erhält, sind die von Hopf ${ }^{12}$ ) angegebenen Sphärenfaserungen; der zugehörige Zerlegungsraum $Z$ ist homöomorph dem ,, $(k-1)$-dimensionalen projektiven $\mathfrak{S}_{q}$-Raum" $P_{k-1}\left(\mathfrak{S}_{q}\right)$ (den man geradezu so definieren kann), also insbesondere für $k=2$ der Sphäre $S^{q}$. In abgekürzter Bezeichnungsweise (vgl. Nr. 2d) können wir diese Zerlegung durch $S^{k q-1} / S^{q-1}=P_{k-1}\left(\Im_{q}\right)$ beschreiben.

g) Die Stiefel'schen Mannigfaltigkeiten $\left.{ }^{14}\right) V_{n, m}$ lassen sich in naheliegender Weise zerlegen.

$V_{n, m}$ ist definiert als Menge aller in einem festen Punkt 0 des $R^{n}$ angreifenden geordneten und normierten Orthogonalsysteme $\sigma_{n, m}$ von $m<n$ Vektoren $\left(\mathfrak{a}_{1}, \ldots, \mathfrak{a}_{m}\right)$ des $R^{n}$, kurz $m$-Systeme genannt (die, in natürlicher Weise topologisiert, zu einer geschlossenen Mannigfaltigkeit wird). Fassen wir nun immer diejenigen Systeme $\sigma_{n, m}$, die in den ersten $k<m$ Vektoren übereinstimmen, zu einer Teilmenge $\bar{A}$ der $V_{n, m}$ zusammen, so erhalten wir eine stetige Zerlegung $3^{\prime}$ der $V_{n, m}$ in Mannigfaltigkeiten $V_{n-k, m-k}$; der Zerlegungsraum $Z$ ist eine $V_{n, k}$ :

$$
3^{\prime}: \quad V_{n, m} / V_{n-k, m-k}=V_{n, k} .
$$

Nachweis der Retrahierbarkeit: Für zwei Punkte $A, B \in V_{n, k}$, d. h. $A=\left(\mathfrak{a}_{1}, \ldots, \mathfrak{a}_{k}\right)$ und $B=\left(\mathfrak{b}_{1}, \ldots, \mathfrak{b}_{k}\right)$ bilden wir die Determinante

$$
G(A, B)=\left|\left(\mathfrak{a}_{i} \cdot \mathfrak{b}_{j}\right)\right|_{k} ;
$$

sie hängt gleichmäßig stetig von $A$ und $B \in V_{n, k} \mathrm{ab} ; G(A, A)=1$, also gibt es eine Zahl $r>0$, so daß

$$
G(A, B) \neq 0 \text { ist für } \varrho(A, B)<r .
$$

12) H. Hopf, Über die Abbildungen von Sphären auf Sphären niedrigerer Dimension (Fund. math. XXV [1935], 427-440), bes. S. $438 \mathrm{ff}$.

13) Einen andern Beweis der Retrahierbarkeit für den Fall $q=2$, der sich ähnlich auch bei $q=4$ (mit Hilfe der Quaternionen) durchführen läßt, haben Hopf und Rueff (Comm. math. helv. 11 [1938], S. 58) angegeben.

14) E. Stiefel, Richtungsfelder und Fernparallelismus im n-dim. Mannigfaltigkeiten (Comm. math. helv. 8 [1935], 3-51), bes. S. $8 \mathrm{ff}$. 
Ist nun $b$ ein Punkt von $\bar{B} \subset V_{n, m}$, d. h. $b=\left(\mathfrak{b}_{1}, \ldots, \mathfrak{b}_{k}, \mathfrak{b}_{k+1} \ldots, \mathfrak{b}_{m}\right)$, so sind für $\varrho(A, B)<r$ die $m$ Vektoren $\mathfrak{a}_{1}, \ldots, \mathfrak{a}_{k}, \mathfrak{b}_{k+1}, \ldots, \mathfrak{b}_{m}$ linear unabhängig; denn aus

folgt

$$
\sum_{i=1}^{k} \lambda_{i} \mathrm{a}_{i}+\sum_{i=k+1}^{m} \mu_{i} \mathrm{~b}_{i}=0
$$

$$
\sum_{i=1}^{k} \lambda_{i}\left(\mathfrak{a}_{i} \cdot \mathfrak{b}_{j}\right)=0, \quad j=1, \ldots, k,
$$

also sind wegen $G(A, B) \neq 0$ alle $\lambda_{i}=0$, also auch alle $\mu_{i}=0$. Man kann also durch Orthogonalisieren den $m$ Vektoren $\mathfrak{a}_{1}, \ldots, \mathfrak{a}_{k}$, $\mathfrak{b}_{k+1}, \ldots, \mathfrak{b}_{m}$ auf eindeutige und stetige Weise einen Punkt $a=\left(\mathfrak{a}_{1}, \ldots, \mathfrak{a}_{k}\right.$, $\left.\mathfrak{a}_{k+1}, \ldots, \mathfrak{a}_{m}\right) \in \bar{A}$ von $V_{n, m}$ zuordnen; dadurch ist eine Abbildung $Q(A, b)$ für $\varrho(A, B)<r$ und $b \in \bar{B}$ definiert, die alle in der Definition erwähnten Bedingungen erfüllt.

h) Eine andere Zerlegung $3^{\prime \prime}$ der Mannigfaltigkeit $V_{n, m}$ erhält man, wenn man immer diejenigen Systeme $\sigma_{n, m}$ zu einer Teilmenge zusammenfaßt, die in derselben $m$-dimensionalen Ebene $E^{m}$ liegen und darin dieselbe Orientierung bestimmen; der Zerlegungsraum $Z$ ist homöomorph dem Büschel aller orientierten $m$-dimensionalen Ebenen $E^{m}$ des $R^{n}$ durch $0, E_{n, m}$; die Elemente dieser Zerlegung sind Mannigfaltigkeiten $V_{m, m-1}$ (denn ein System $\sigma_{n, m}$, das in einer festen Ebene $E^{m}$ liegt und darin eine vorgegebene Orientierung bestimmt, ist schon durch die $(m-1)$ ersten Vektoren eindeutig festgelegt):

$$
3^{\prime \prime}: \quad V_{n, m} / V_{m, m-1}=E_{n, m} \text {. }
$$

Für zwei Punkte $a, b \in V_{n, m}, a=\left(\mathfrak{a}_{1}, \ldots, \mathfrak{a}_{m}\right)$ und $b=\left(\mathfrak{b}_{1}, \ldots, \mathfrak{b}_{m}\right)$ ist die Determinante

$$
G(\boldsymbol{a}, \boldsymbol{b})=\left|\left(\mathfrak{a}_{i} \cdot \mathfrak{b}_{j}\right)\right|_{m},
$$

wie leicht zu sehen, nur abhängig von den orientierten Ebenen $A=P a$ und $B=P b$. Insbesondere ist $G(a, a)=1$, also gibt es eine Zahl $r>0$, so $\operatorname{da} B G(a, b) \neq 0$ ist, wenn $\varrho(A, B)<r . \mathfrak{b}_{1}^{\prime}, \ldots, \mathfrak{b}_{m}^{\prime}$ seien die Vektoren, die durch Normalprojektion von $\mathfrak{b}_{1}, \ldots, \mathfrak{b}_{m}$ auf die Ebene $A$ entstehen; dann ist $\mathfrak{a}_{i} \cdot \mathfrak{b}_{j}=\mathfrak{a}_{i} \cdot \mathfrak{b}_{j}^{\prime}$ und aus $\sum_{j=1}^{m} \lambda_{j} \mathfrak{b}_{i}^{\prime}=0$ folgt wegen $\sum_{j=1}^{m} \lambda_{j}\left(\mathfrak{a}_{i} \cdot \mathfrak{b}_{j}^{\prime}\right)=0$ $(i=1, \ldots, m)$, daß alle $\lambda_{j}=0$ sind. Die $\mathfrak{b}_{j}^{\prime}$ sind also linear unabhängig und ergeben, in bestimmter Reihenfolge orthogonalisiert, ein System 
$\sigma_{n, m}$ in der Ebene $A$, d. h. einen Punkt $a^{\prime} \in \bar{A}$;

$$
a^{\prime}=Q(A, b)
$$

ist die Retraktionsabbildung der Zerlegung.

g) Man kann diese Zerlegungen $3^{\prime}$ und $3^{\prime \prime}$ der $V_{n, m}$ kombinieren: Man faßt alle diejenigen Systeme $\sigma_{n, m}$ zu einer Teilmenge zusammen, die in den ersten $k<m$ Vektoren übereinstimmen und bei denen die letzten $m-k$ Vektoren dieselbe $(m-k)$-dimensionale orientierte Ebene bestimmen. Auch diese Zerlegung ist, wie man leicht feststellt, retrahierbar; ihr Zerlegungsraum ${ }^{15}$ ) gestattet seinerseits eine Zerlegung, bei welcher der Zerlegungsraum eine $V_{n, k}$ und die Elemente Ebenenbüschel $E_{n-k, m-k}$ sind.

\section{$\S 2$. Abbildungssätze}

\section{Vorbereitendes. Ein Lemma}

a) 3 sei eine retrahierbare Überdeckung eines Kompaktums $R, Z$ der zugehörige Utberdeckungsraum und $Q(A, b)$ die in der Definition (Nr. 1c) genannte Abbildung; $X$ sei ein Kompaktum. $Q(A, b)$ induziert in folgender Weise eine Abbildung zwischen den Abbildungsräumen $R^{X}$ und $Z^{X}: F$ und $G$ seien zwei Abbildungen aus $Z^{X}$ mit $\varrho(F, G)<r$, und $F$ sei Spur von $f \in R^{X}$ (Nr. 1d), also $f(x) \in \widetilde{F}(x)$ für alle $x \in X$; dann verstehen wir unter $g=Q(G, f)$ das durch

$$
g(x)=Q(G(x), f(x)), \quad x \in X
$$

erklärte Element von $R^{X}$. Für dieses gilt $G=S p g$. In $Z^{x}$ besteht also die ganze $r$-Umgebung einer Spur $F$ aus Spuren, und durch $Q(G, f)$ wird bei festem $f$ eine Abbildung von $U(F, r) \subset Z^{X}$ in $R^{X}$ mit der "Anfangsbedingung" $Q(F, f)=f$ definiert, derart, daß immer

ist.

$$
G=S p Q(G, f)
$$

Diese Abbildung ist stetig. Beweis: Es sei $g=Q(G, f)$ und $g^{\prime}=Q\left(G^{\prime}, f\right)$. Dann ist

$$
\varrho\left(g(x), g^{\prime}(x)\right)=\varrho\left[Q(G(x), f(x)), Q\left(G^{\prime}(x), f(x)\right)\right]
$$

wegen der gleichmäßigen Stetigkeit der Abbildung $Q(A, b)$ (s. Nr. 1c)

15) Ein Beispiel eines solchen Raumes ist ( $k=1, m=3$ ) die Mannigfaltigkeit der an die $S^{n-1}$ tangentialen orientierten Flächenelemente (vgl. Nr. 17). 
kleiner als eine beliebige Zahl $\varepsilon>0$, wenn $\varrho\left(G(x), G^{\prime}(x)\right)<\delta(\varepsilon)$, wobei $\delta$ von $x$ nicht abhängt; also ist $\varrho\left(g, g^{\prime}\right)<\varepsilon$, wenn $\varrho\left(G, G^{\prime}\right)<\delta$.

b) Aus den Eigenschaften von $Q(A, b)$ folgt ferner für das eben erklärte Element $g=Q(G, f) \in R^{X}$ :

Wenn, für einen bestimmten Punkt $x_{0}$ von $X, G\left(x_{0}\right)=F\left(x_{0}\right)$ ist, dann ist auch $g\left(x_{0}\right)=f\left(x_{0}\right)$.

Beweis : $g\left(x_{0}\right)=Q\left(G\left(x_{0}\right), f\left(x_{0}\right)\right)=Q\left(F\left(x_{0}\right), f\left(x_{0}\right)\right)=f\left(x_{0}\right)$.-

Wenn für die Teilmengen $X_{0} \subset X$ und $Z_{0} \subset Z$ gilt $G\left(X_{0}\right) \subset Z_{0}$, dann ist $g\left(X_{0}\right) \subset R_{0}$, wo $R_{0}=\sum_{A \in Z_{0}} \bar{A}$.

Beweis: Für alle $x \in X_{0}$ ist $g(x)=Q(G(x), f(x)) \in \overline{G(x)} \subset R_{0}$.

c) Deformationen: Eine Deformation einer Abbildung $f \in Y^{X}$ ( $Y$ sei ein beliebiger metrischer Raum) ist gleichbedeutend mit einem Weg $\alpha$ in $Y^{X}$, der im Punkte $f$ beginnt, d. h. einer stetigen Abbildung $\alpha(t)$ der Einheitsstrecke $0 \leqslant t \leqslant 1$ in $Y^{\boldsymbol{X}}$ mit $\alpha(0)=f$. Zwei Abbildungen $f$ und $g$ heißen homotop, wenn es in $Y^{X}$ einen Weg $\alpha$ gibt mit $\alpha(0)=f$ und $\alpha(1)=g$. Man kann diesen Homotopiebegriff verschärfen, indem man verlangt, daß bei der ganzen Deformation $\alpha$ jede der Abbildungen $\alpha(t)(0 \leqslant t \leqslant 1)$ einen Punkt $x_{0} \in X$ auf einen vorgegebenen Punkt $y_{0} \in Y$, oder eine Teilmenge $X_{0} \subset X$ in eine Teilmenge $Y_{0} \subset Y$ abbildet, d. h. indem man nur Wege $\alpha$ auf einen Teilraum $\left(Y^{\boldsymbol{X}}\right)_{0}$ von $Y^{\boldsymbol{X}}$ zuläßt; man muß dann genauer sagen, $f$ und $g$ seien ,homotop in $\left(Y^{X}\right)_{0}$ “.

Eine Abbildung $f \in Y^{\boldsymbol{X}}$, die (in $Y^{\boldsymbol{X}}$ ) einer Abbildung auf einen einzigen Punkt von $Y$ homotop ist, heißt zusammenziehbar (in $Y^{X}$ ); eine Abbildung $f \in Y^{X}$ heißt wesentlich, wenn für jede zu ihr homotope Abbildung $g$ die Bildmenge $g(X)=Y$ ist.

Eine (kompakte) Teilmenge $M \subset Y$ heißt zusammenziehbar in $Y$, wenn die identische Abbildung von $M$ auf sich in $Y^{M}$ zusammenziehbar ist. Ein Kompaktum $Y$ heißt wesentlich auf sich, wenn die identische Abbildung von $Y$ auf sich in $Y^{Y}$ wesentlich ist.

Ist $\gamma$ in der Überdeckung 3 ein Weg in $R^{X}, \Gamma$ ein Weg in $Z^{X}$ und für $0 \leqslant t \leqslant 1 \Gamma(t)=S p \gamma(t)$, so nennen wir $\Gamma$ eine Spur von $\gamma$ (und sagen auch, die Deformation $\Gamma$ sei eine Spur).

d) Lemma: Wenn der Anfangspunkt eines Weges $\Gamma$ in $Z^{X}$ eine Spur ist, so ist der ganze Weg $\Gamma$ eine Spur. Genauer: Wenn $\Gamma(0)=F=S p t$, so gibt es einen Weg $\gamma$ in $R^{X}$ mit $\gamma(0)=f$, derart, daß $\Gamma=S p \gamma$.

Anders formuliert: Jede zu einer Spur $F \in Z^{X}$ homotope Abbildung $G \in Z^{X}$ ist eine Spur. Genauer: Wenn $F=S p f$, und $G$ zu $F$ homotop ist, 
dann ist $G$ Spur einer zu $f$ homotopen Abbildung $g \in R^{X}$, und die ganze Deformation von $F$ ist Spur der Deformation von $f$.

Beweis: Der Weg $\Gamma$ ist gegeben durch $\Gamma(t) \in Z^{X}, 0 \leqslant t \leqslant 1$, mit $\Gamma(0)=F=S p f$. Man kann eine Zahl $\delta>0$ sowählen, daß für $\left|t-t^{\prime}\right|<\delta$ gilt

Dann seien

$$
\varrho\left(\Gamma(t), \Gamma\left(t^{\prime}\right)\right)<r
$$

$n+1$ Zahlen mit

$$
0=t_{0}<t_{1}<\cdots<t_{n}=1
$$

Wir setzen nun

$$
t_{i}-t_{i-1}<\delta, i=1, \ldots, n \text {. }
$$

und

$$
\gamma(0)=f
$$

$$
\gamma(t)=Q\left(\Gamma(t), \gamma\left(t_{i-1}\right)\right) \text { für } t_{i-1} \leqslant t \leqslant t_{i}, i=1, \ldots, n .
$$

Dadurch ist ein Weg $\gamma$ in $R^{X}$ definiert, der in $f$ beginnt, und für welchen gilt

$$
\Gamma(t)=S p \gamma(t) \quad(0 \leqslant t \leqslant 1) ;
$$

die Existenz eines solchen Weges war aber gerade nachzuweisen.

e) Aus dem in Abschnitt b) dieser Nummer Gesagten ersieht man, daß das Lemma und seine Konsequenzen (Nr. 4) auch gültig bleiben, wenn man allen betrachteten Abbildungen von $X$ in $R$ und $Z$ eine oder mehrere Bedingungen der folgenden Typen 1 und 2 auferlegt (d. h. sich auf einen gewissen Teilraum von $Z^{X}$ und einen zugehörigen Teilraum von $R^{X}$ beschränkt):

1. $x_{0}, a_{0}, A_{0}$ seien vorgeschriebene Punkte bzw. von $X, R, Z$, mit $a_{0} \in \bar{A}_{0}$. Es werden nur solche Abbildungen $f \in R^{X}$ und $F \in Z^{X}$ zugelassen, bei welchen $f\left(x_{0}\right)=a_{0}$ bzw. $F\left(x_{0}\right)=A_{0}$ ist.

2. $X_{0}$ und $Z_{0}$ seien Teilmengen von $X$ bzw. $Z$, und $R_{0}=\sum_{A \in Z_{0}} \bar{A} \subset R$. Es werden nur Abbildungen $f$ und $F$ zugelassen, bei welchen $f\left(X_{0}\right) \subset R_{0}$ bzw. $F\left(X_{0}\right) \subset Z_{0}$ ist.

f) Wir nehmen nun speziell an, 3 sei eine Zerlegung. Dann bewirkt die Projektion $P$ (s. Nr. lf) von $R$ auf den Zerlegungsraum $Z$ eine Abbildung von $R^{X}$ in $Z^{X}$, die dem Punkt $f \in R^{X}$ den Punkt

$$
F=P f \in Z^{X}
$$

zuordnet und die wir ebenfalls mit $P$ bezeichnen; wegen der gleichmäßigen Stetigkeit der Projektion ist sie selbst stetig. 
Wenn $R$ (und infolgedessen auch $Z$, wie man mit Hilfe der Abbildungen $P$ und $Q$ leicht zeigen kann) zusammenhängend und lokal zusammenziehbar ${ }^{16}$ ) ist, und wenn das Kompaktum $X$ endlich-dimensional ist, dann sind nach Hurewicz ${ }^{17}$ ) die Komponenten von $R^{X}$ (bzw. $Z^{X}$ ) offene, durch stetige Wege zusammenhängende Mengen, also identisch mit den Klassen homotoper Abbildungen von $X$ in $R$ (bzw. $Z$ ). Wir bezeichnen die Abbildungsklasse, zu welcher $f$ gehört, mit $\{f\}$ (oder wenn kein Mißverständnis möglich ist, ebenfalls mit $f$ ).

Da die Abbildung $P$ von $R^{X}$ in $Z^{X}$ stetig ist, bildet sie jede Komponente $\{f\} \subset R^{X}$ in die Komponente $\{P f\} \subset Z^{X}$ ab; nach dem Lemma ist aber jede Abbildung $G$ aus der Klasse $\{P f\}$ Spur einer zu $f$ homotopen Abbildung $g$, d. h. $G=P g ; P$ bildet also $\{f\}$ auf $\{P f\}$ ab, und man kann für eine Zerlegung 3 dem Lemma die kurze Form geben $\left.{ }^{18}\right)$ :

$$
P\{f\}=\{P f\} .
$$

g) Wir führen zur bequemern Formulierung der folgenden Sätze noch eine Bezeichnung ein: Wenn 3 eine Überdeckung von $R$ ist, so soll eine Abbildung $f \in R^{X^{X}}$ relativ-zusammenziehbar (bezüglich 3) heißen, wenn sie einer Abbildung $g$ homotop ist, bei welcher die Bildmenge $g(X)$ ganz in einem Element $\bar{A}$ der Überdeckung $\mathcal{B}$ enthalten ist. Eine Teilmenge $M \subset R$ heißt relativ-zusammenziehbar, wenn die identische Abbildung von $M$ auf sich (als Abbildung aus $R^{M}$ ) relativ-zusammenziehbar ist.

\section{Hauptsätze}

3 sei eine retrahierbare Überdeckung des Kompaktums $R, Z$ der zugehörige Überdeckungsraum, $X$ ein beliebiges Kompaktum.

Satz A: Jede zusammenziehbare Abbildung $F \in Z^{X}$ ist eine Spur.

Satz B: Wenn eine Abbildung $f \in R^{X}$ eine zusammenziehbare Spur besitzt, dann ist $f$ relativ-zusammenziehbar bezüglich 3 .

Der Beweis von Satz A und Satz B ergibt sich direkt aus dem Lemma (Nr. 3d) und der Bemerkung, daß eine Abbildung $G$ von $X$ auf einen Punkt $A \in Z$ Spur einer Abbildung $f \in \bar{A}^{X}$ ist.

Korollar zu Satz $A$ : Wenn $X$ in sich zusammenziehbar ist, so ist jede Abbildung $F^{T} \in Z^{X}$ eine Spur.

Korollar $z u$ Satz $B$ : Wenn 3 eine Zerlegung von $R$ ist, und $P$ die Projektion von $R$ auf $Z$, so ist eine Abbildung $f \in R^{X}$ dann und nur dann relativ-zusammenziehbar, wenn $P f \in Z^{X}$ zusammenziehbar ist (,dann“

\footnotetext{
${ }^{16}$ ) das bedeutet: jede Umgebung $U$ enthält eine in $U$ zusammenziehbare Umgebung.

17) s. $\left.{ }^{8}\right)$, S. 113.

18) Vgl. für Gruppenzerlegungen: Hurewiez ${ }^{8}$ ), S. 117, Satz VIII.
} 
folgt aus Satz B, ,nur dann“ aus der Stetigkeit der Abbildung $P$ von $R^{X}$ in $Z^{X}$ ).

Satz $C:$ Wenn 3 eine Zerlegung von $R$ ist, so ist die Spur einer wesentlichen Abbildung $f \in R^{X}$ wesentlich.

Beweis: $f$ besitzt genau eine Spur, nämlich $F=P f$; wenn $F$ einer Abbildung $G$ homotop ist, bei welcher $A \in Z$ nicht zur Bildmenge $G(X)$ gehört, so ist nach dem Lemma $f$ einer Abbildung $g$ (mit $G=P g$ ) homotop, bei welcher $\bar{A} \subset R$ nicht zur Bildmenge $g(X)$ gehört. Bei einer wesentlichen Abbildung $f$ ist das nicht möglich.

Korollar $z u$ Satz $C$ : Wenn 3 eine Zerlegung von $R$ ist, und wenn $R$ auf sich wesentlich ist, dann ist die Projektion $P$ von $R$ auf $Z$ eine wesentliche Abbildung (also muß auch $Z$ auf sich wesentlich sein).

Satz D: Die Überdeckung 3 von $R$ enthalte eine Zerlegung $\mathcal{Z}^{\prime}$ (Definition s. Nr. 2f) mit dem Zerlegungsraum $Z^{\prime} \subset Z$. Wenn $R$ auf sich wesentlich ist, so ist die Projektion $P$ von $R$ auf $Z^{\prime}$ in $Z^{R}$ nicht zusammenziehbar, also $Z^{\prime}$ in $Z$ nicht zusammenziehbar.

Beweis: Es genügt vorauszusetzen, daß $R$ bezüglich $3^{\prime}$ nicht relativzusammenziehbar ist; dann folgt die Behauptung direkt aus Satz B, weil $P$ eine Spur der Identität von $R$ ist. - Wenn man voraussetzt, daß $R$ auf sich wesentlich ist, so kann man die Behauptung verschärfen: $P$ läßt sich in $Z^{R}$ nicht in eine Abbildung $F \in Z^{\prime R}$ deformieren, bei welcher die Bildmenge $F(R)$ eine echte Teilmenge von $Z^{\prime}$ ist; also auch: $Z^{\prime}$ läßt sich in $Z$ nicht in eine echte Teilmenge von sich deformieren.

\section{Anwendungen}

a) Satz 1: Bei einer regulären Faserung (Nr. 2d) der geschlossenen Mannigfaltigkeit $R$ ist die Faserabbildung eine wesentliche Abbildung von $R$ auf den Faserraum.

Beweis: Eine geschlossene Mannigfaltigkeit ist auf sich wesentlich ${ }^{10}$ ); die Behauptung folgt also direkt aus dem Korollar zu Satz C.

Ein Korollar zu Satz 1 ist der von Hurewicz ${ }^{20}$ ) bewiesene Satz, daß eine geschlossene Lie'sche Gruppe sich wesentlich auf ihre Wirkungsräume abbilden läßt.

b) Wir können den Satz D auf die in Nr. $2 e, f$ beschriebenen Überdeckungen der Sphäre $S^{n-1}$ mit Groß-Sphären $S^{q-1}$ anwenden: Für $n=k q(q=1,2,4 ; k=2,3, \ldots$ und $q=8 ; k=2)$ enthält diese Überdeckung eine Zerlegung mit dem Zerlegungsraum $P_{k-1}\left(\Im_{a}\right)$ und es gilt

19) s. ${ }^{1}$ ), S. 519, Satz II.

20) s. ${ }^{8}$ ), S. 118 , oben. 
Satz 2: Der projektive $\mathfrak{S}_{q}$-Raum $P_{k-1}\left(\Im_{q}\right)(q=1,2,4 ; k=2,3, \ldots$ und $q=8 ; k=2$ ) ist im Großkreisraum $G_{k q-1, q-1}$ nicht zusammenziehbar.

Insbesondere gibt es in $G_{2 q-1, q-1}$ ein topologisches, nicht zusammenziehbares Bild der $\mathcal{S}^{q}$.

c) Die Anwendung von Satz C auf die Hopf'schen Sphärenfaserungen (s. Nr. 2f)

$$
S^{k q-1} / S^{q-1}=P_{k-1}\left(\Im_{q}\right), q=1,2,4 ; k=2,3, \ldots \text { und } q=8 ; \mathrm{k}=2
$$
ergibt:

Satz 3: Ein Kompaltum $X$, das sich wesentlich auf $S^{k a-1}$ abbilden läßt, läßt sich auch wesentlich auf $P_{k-1}\left(\mathfrak{S}_{q}\right)$ abbilden.

Korollar: Es gibt eine wesentliche Abbildung der $S^{k q-1}$ auf $P_{k-1}\left(\Im_{q}\right)$. Beschränken wir uns auf $k=2$, so können wir $P_{1}\left(\Im_{q}\right)$ durch die damit homöomorphe $S^{q}$ ersetzen und erhalten (wenn wir den uninteressanten Fall $q=1$ weglassen):

Satz $3^{\prime}$ : Für $q=2,4,8$ läßt sich ein Kompaktum $X$, das man wesentlich auf $S^{2 q-1}$ abbilden kann, auch auf $S^{q}$ wesentlich abbilden.

Korollar: Für $q=2,4,8$ gibt es eine wesentliche Abbildung der $S^{2 q-1}$ auf die $S^{q}$.

Diese letzte Aussage ist in einem allgemeinern Satze von Hopf ${ }^{21}$ ) enthalten (der besagt, daß sie für alle geraden $q$ gilt); es ist aber bemerkenswert, daß nach unserer Methode ${ }^{22}$ ) der Beweis der Wesentlichkeit ohne Heranziehung von Homologiebegriffen geführt wird.

Wir machen noch Gebrauch von folgendem Resultate aus der Freudenthal'schen Theorie der Sphärenabbildungen ${ }^{23}$ ): Die $S^{N}$ läßt sich wesentlich auf die $S^{n}$ abbilden, (u. a.) wenn $N-n=1,3,7$ und $n \geqslant N-n+2$ ist. Es gibt also wesentliche Abbildungen der $S^{N}$ auf die $S^{k q-1}$ für $N=k q$, $k q+2, k q+6$, falls diese Zahlen kleiner sind als $2 k q-3$, und nach Satz 3 infolgedessen auch wesentliche Abbildungen der $S^{N}$ auf $P_{k-1}\left(\mathcal{S}_{q}\right)$. Setzen wir wieder speziell $k=2$ (wobei wir $q=1$ und $q=2$ weglassen), so erhalten wir

Satz 4: Für $q=4$ und $N=8,10$, ferner für $q=8$ und $N=16,18,22$ gibt es wesentliche Abbildungen der $S^{N}$ auf die $S^{q}$.

d) Mit Hilfe der in Nr. 2e beschriebenen Zerlegung der Stiefel'schen Mannigfaltigkeit $V_{n, m}$

$$
V_{n, m} / V_{n-k, m-k}=V_{n, k}, \quad 0<k<m<n,
$$

21) s. ${ }^{12}$ ), S. 431, Satz II.

22) nach welcher Hurewicz (vgl. ${ }^{8}$ )) den Fall $q=2$ (unter Benützung der Tatsache, daß $S^{3}$ Gruppenmannigfaltigkeit ist!) behandelt hat.

23) $H$. Freudenthal, Úber die Klassen der Sphärenabbild ungen I (Comp. math.V, 1937, 299-314), bes. S. 301. 
worin wir speziell $m=n-1$ setzen, können wir in einfacher Weise folgenden Satz von Wazewski ${ }^{24}$ ) beweisen:

Satz 5 (Satz von Wazewski): $X$ sei eine Vollkugel (beliebiger Dimension), $B$ eine Matrix von $n$ Spalten und $k<n$ Zeilen, deren Elemente reelle stetige Funktionen von $x \in X$ sind, und die in jedem Punkte $x \in X$ den Rang $k$ hat. Dann kann man $B$ zu einer $n$-reihigen quadratischen Matrix ergänzen, deren Elemente stetige Funktionen von $x \in X$ sind, und die in jedem Punkt $x \in X$ nicht-singulär ist.

Beweis: Es genügt offenbar, den Fall zu behandeln, wo $B$ orthogonal ist, d. h. ihre $k$ Zeilenvektoren ein System $\sigma_{n, k}$ bilden und zu zeigen, daß man dieses System zu einem System $\sigma_{n, n-1}$ ergänzen kann, das stetig von $x \in X$ abhängt (denn man kann die Vektoren dieses Systems $\sigma_{n, n-1}$ als Zeilenvektoren einer orthogonalen Matrix auffassen und diese Matrix in eindeutiger Weise zu einer quadratischen orthogonalen mit der Determinante +1 ergänzen). Mit andern Worten: es genügt zu zeigen, daß die durch $B$ gegebene Abbildung von $X$ in $V_{n, k}$ bezüglich der oben genannten Zerlegung von $V_{n, n-1}$ eine Spur ist. Das ist aber nach dem Korollar zu Satz A (s. Nr. 4) der Fall, weil $X$ in sich zusammenziehbar ist.

\section{§ 3. Homotopiegruppen bei retrahierbaren Zerlegungen}

\section{Die Hurewicz'schen Formeln}

a) Voraussetzungen: 3 sei eine retrahierbare Zerlegung des Kompaktums $R, Z$ der zugehörige Zerlegungsraum. $R$ sei zusammenhängend und lokal zusammenziehbar; dann gilt dasselbe auch für $Z$. (Vgl. Nr. 3 f.) $\bar{A} \subset R$ sei ein Element der Zerlegung, das (in sich) lokal zusammenziehbar ist. Wir zeichnen in $Z$ den Punkt $A$, in $R$ einen Punkt $a \in \bar{A}$ für die Bildung der Homotopiegruppen ${ }^{25}$ ) aus; das besagt: von jeder im folgenden betrachteten Abbildung einer Sphäre in $R, \bar{A}$ oder $Z$ wird verlangt, da $B$ dabei ein fester Punkt $\dot{x}_{0}$ dieser Sphäre in den Punkt $a$ bzw. $A$ übergeht.

b) Unter diesen Voraussetzungen sind die Homotopiegruppen der Räume $R, \bar{A}$ und $Z$ von höchstens abzählbarer Ordnung und handeln von Eigenschaften der Rüume im Großen; die Elemente der $n$-ten Homotopiegruppe von $R$ sind (vgl. Nr. 3f) die Komponenten von $R s^{n}$, d. h. die Klassen hcmotoper Abbildungen der $S^{n}$ in $R$,unter Festhaltung

24) T. Wazewski, Sur les matrices dont les éléments sont des fonetions continues (Comp. math. II [1935], 63-68), bes. S. 63).

${ }^{25}$ ) Wegen der Definition der Homotopiegruppen vgl. man: Hurewic ${ }^{8}$ ), S. 114; Freuden thal $\left.{ }^{23}\right)$, S. 302 . 
von $a^{" ~(d a s ~ E n t s p r e c h e n d e ~ g i l t ~ f u ̈ r ~} \pi_{n}(\bar{A})$ und $\pi_{n}(Z)$ ); die Klasse der zusammenziehbaren Abbildungen ist das Neutralelement der Gruppe, das wir mit 0 bezeichnen ${ }^{26}$ ).

Die Klassen der bezüglich 3 relativ-zusammenziehbaren (s. Nr. 3g) Abbildungen, $d . h$. die Komponenten von $R^{S n}$, die Abbildungen aus $\bar{A}^{S n}$ enthalten, bilden eine Untergruppe von $\pi_{n}(R)$, die wir mit $\varphi_{n}(3)$ bezeichnen. Ferner bilden die Klassen derjenigen Abbildungen aus $\bar{A}^{\text {Sn }}$, die in $R^{S n}$ zusammenziehbar sind, eine Untergruppe $\psi_{n}(\boldsymbol{Z})$ von $\pi_{n}(\bar{A})$. Wir werden bei Faserungen $R / F=Z$ statt $\varphi_{n}(3)$ und $\psi_{n}(3)$ auch $\varphi_{n}(R / F)$ und $\psi_{n}(R / F)$ schreiben. - Offenbar ist $\varphi_{n}(3)$ isomorph der Faktorgruppe von $\left.\pi_{n}(\bar{A}) \operatorname{nach} \psi_{n}(3)^{27}\right)$

$$
\varphi_{n}(3) \approx \pi_{n}(\bar{A}) / \psi_{n}(3) .
$$

c) Aus der Definition der Summe $\left.{ }^{25}\right) f+g$ zweier Abbildungen $f$ und $g \in R S^{n}$ ersieht man direkt, daß für die Projektion $P$ von $R$ auf $Z$ gilt:

$$
P(f+g)=P f+P g .
$$

Die Projektion $P$, die jede Komponente von $R S^{n}$ auf eine solche von $Z s^{n}$ abbildet, bewirkt also einen Homomorphismus von $\pi_{n}(R)$ in $\pi_{n}(Z)$

$$
\left.\pi_{n}(R) \sim P \pi_{n}(R) \subset \pi_{n}(Z)^{27}\right)
$$

dessen Kern (darunter verstehen wir wie üblich die Untergruppe, die bei dem Homomorphismus auf die 0 der Bildgruppe abgebildet wird) gemäß dem Korollar zu Satz B (Nr.4) genau aus den Klassen der relativzusammenziehbaren Abbildungen besteht:

$$
P \pi_{n}(R) \approx \pi_{n}(R) / \varphi_{n}(3)
$$

d) $V^{n}$ sei eine $n$-dimensionale Vollkugel, $\sum^{n-1}$ ihre Randsphäre $(n>0)$. Wir bezeichnen mit $R^{v^{n}}$ den Raum derjenigen Abbildungen $f$ von $V^{n}$ in $R$, bei denen $\Sigma^{n-1}$ in $\bar{A}$ abgebildet wird (unter Beachtung der in Nr. 6a genannten Bedingungen); die zu $f$ gehörige Abbildung der $\sum^{n-1}$ in $\bar{A}$ bezeichnen wir mit $r f$ (als Abbildung $f^{\prime} \in R \Sigma^{n-1}$ aufgefaßt, ist sie in $R^{\Sigma^{n-1}}$ zusammenziehbar !). Für $n \geqslant 2$ definieren wir die Summe $f+g$ zweier Abbildungen $f, g \in R^{n}$, indem wir die auf $\sum^{n-1}$ für $f^{\prime}=r f$ und

28) Vgl. ${ }^{25}$ ). Für $n \geq 2$ sind die Homotopiegruppen abelsch; wir bezeichnen aber auch für $n=1$ (Fundamentalgruppe) die Gruppenoperation als Addition.

27) $\approx$ isomorph, - homomorph. 
$g^{\prime}=r g$ nach der Hurewicz'schen Vorschrift erklärte Addition ins Innere der $V^{n}$ fortsetzen; dabei ist also

$$
r(f+g)=r f+r g .
$$

Vermöge dieser Addition werden die Komponenten von $R^{V^{n}} \mathrm{zu}$ Elementen einer Gruppe $v_{n}(\mathfrak{Z})$.

$V$ sei eine stetige Abbildung der $V^{n}$ auf die $S^{n}$, welche $\sum^{n-1}$ auf den Punkt $x_{0} \in S^{n}$ und das Innere der $V^{n}$ topologisch auf $S^{n}-x_{0}$ abbildet. Durch Vermittlung von $V$ können wir die Abbildungen der $S^{n}$ in $Z$ eineindeutig denjenigen Abbildungen von $V^{n}$ in $Z$ zuordnen, bei welchen $\sum^{n-1}$ auf den Punkt $A \in Z$ abgebildet wird, d. h. $\pi_{n}(Z)$ auch als Gruppe der Klassen solcher Abbildungen $F$ der $V^{n}$ in $Z$ auffassen; mit $Z^{n}$ werde immer nur der Raum dieser Abbildungen bezeichnet. Nach dem Korollar zu Satz A (Nr. 4) ist jede Abbildung $F \in Z^{V^{n}}$ eine Spur (man beachte das in Nr. 3e Gesagte), und zwar einer Abbildung $f \in R{ }^{n}$. Umgekehrt ist die Spur $P f$ jeder Abbildung aus $R V^{n}$ eine Abbildung aus $Z \nabla^{n}$. $P$ bildet also $v_{n}(3)$ auf $\pi_{n}(Z) \mathrm{ab}$, und zwar homomorph wegen

$$
P(f+g)=P f+P g .
$$

Der Kern dieses Homomorphismus ist 0, denn jede relativ-zusammenziehbare Abbildung $f \in R^{V^{n}}$ ist zusammenziehbar; somit

$$
v_{n}(3) \approx \pi_{n}(Z)
$$

Es sei noch bemerkt, da $\beta$ in diesem Isomorphismus der Untergruppe $P \pi_{n}(R)$ von $\pi_{n}(Z)$ die Untergruppe derjenigen Komponenten von $R^{v^{n}}$ entspricht, die Abbildungen $f$ mit $f\left(\sum^{n-1}\right)=a$ enthalten; wir bezeichnen diese Untergruppe von $v_{n}(3)$ mit $\mu_{n}(3)$ :

$$
\mu_{n}(3) \approx P \pi_{n}(R)
$$

e) Zu jeder Abbildung $f \in R^{n}$ gehört eine in $R \Sigma^{n-1}$ zusammenziehbare Abbildung $f^{\prime}=r f \in \bar{A}^{\Im^{n-1}}$, und umgekehrt gibt es $\mathrm{zu}$ jeder solchen Abbildung $g^{\prime} \in \bar{A}^{\Sigma^{n-1}}$ eine Abbildung $g \in R \nabla^{n}$ (die ,Zusammenziehnng"“ von $g^{\prime}$ ) mit $g^{\prime}==r g$. Dabei wird jeder Komponente von $R^{v^{n}}$ eine Komponente von $A^{\Sigma^{n-1}}$ zugeordnet, und man erhält wegen (3) einen Homomorphismus von $v_{n}(3)$ in $\pi_{n-1}(\bar{A})$, genauer aut $\psi_{n-1}(3)$; sein Kern besteht aus den Klassen derjenigen Abbildungen $f \in R^{V^{n}}$, für welche $r f$ in $\bar{A} \Sigma^{n^{-1}}$ 
zusammenziehbar ist, d. h. er ist identisch mit der eben genannten Gruppe $\mu_{n}(3):$

$$
v_{n}(3) / \mu_{n}(3) \approx \psi_{n-1}(3)
$$

oder vermöge des durch $P$ bewirkten Isomorphismus (4):

$$
\pi_{n}(Z) / P \pi_{n}(R) \approx \psi_{n-1}(3)
$$

Die Formeln (2) und (7) wollen wir als Hurewicz'sche Formeln bezeichnen; sie sind eine Verallgemeinerung von Ergebnissen, die Hurewicz für Gruppenzerlegungen angegeben hat ${ }^{28}$ ).

Zusammengefaßt:

Satz E: Unter den Voraussetzungen 6a) und mit den Bezeichnungen $6 \mathrm{~b})$ gilt für die Zerlegung 3 von $R$ :

$$
\begin{aligned}
& \pi_{n}(Z) / P \pi_{n}(R) \approx \psi_{n-1}(3), \quad n \geqslant 2 \\
& P \pi_{n}(R) \approx \pi_{n}(R) / \varphi_{n}(3), n \geqslant 1 \\
& \varphi_{n}\left(\mathfrak{3 )} \approx \pi_{n}(\bar{A}) / \psi_{n}(\mathfrak{3}), n \geqslant 1\right.
\end{aligned}
$$

f) Zusatz für den Fall $n=1$ (Fundamentalgruppe):

Wenn $A$ zusammenhängend ist, so gilt für $n=1$ an Stelle von Formel (7)

$$
P_{\pi_{1}}(R)=\pi_{1}(Z)
$$

denn jeder geschlossene Weg in $Z$, d. h. jede Abbildung von $S^{1}$ in $Z$, kann dann als Spur eines geschlossenen Weges in $R$ aufgefaßt werden.

Für den Fall einer Gruppenzerlegung gilt Formel (7) unverändert auch für $n=1$, wenn man unter $\psi_{0}(3)$ die Komponenten-Gruppe der Untergruppe $\bar{A}$ von $R$ versteht, und $P \pi_{1}(R)$ ist dann im Zentrum von $\pi_{1}(Z)$ enthalten. (Man vgl. Hurewicz ${ }^{28}$ ).)

g) Nach $(7)$ ist $(n \geqslant 2) \psi_{n-1}(3)=0$ gleichbedeutend mit

also:

$$
\pi_{n}(Z)=P \pi_{n}(R)
$$

Korollar zu Satz $E$ : Dann und nur dann ist $\psi_{n-1}(3)=0$, wenn jede Abbildung $F \in Z S^{n}$ Spur einer Abbildung $f \in R S^{n}$ ist.

\footnotetext{
28) 8. $\left.{ }^{8}\right)$, S. 118, Satz XII.
} 


\section{Eine Verschärfung}

a) Geometrische Anwendungen legen folgende algebraischen Betrachtungen nahe:

Wir nehmen an, man könne die in der Nummer 6e) betrachtete homomorphe Abbildung $\left(f, f^{\prime}\right.$ etc. bedeuten hier Abbildungsklassen)

$$
f^{\prime}=r f
$$

von $v_{n}(3)$ auf $\psi_{n-1}(3)(n \geqslant 2)$ durch einen Homomorphismus $H$ von $\psi_{n-1}(3)$ in $v_{n}(3)$ umkehren in dem Sinne, daß für jedes $f^{\prime} \epsilon \psi_{n-1}(3)$

$$
r H f^{\prime}=f^{\prime}
$$

ist. Dann ist $H$ ein Isomorphismus (von $\psi_{n-1}(3)$ in $v_{n}(3)$ ); denn aus $H f^{\prime}=0$ folgt $r H f^{\prime}=f^{\prime}=0$. Ferner ist der Durchschnitt $H \psi_{n-1}(3) \cap \mu_{n}(3)$ leer: aus $H f^{\prime} \in \mu_{n}(3)$ folgt

$$
r H f^{\prime}=f^{\prime}=0
$$

also

$$
H f^{\prime}=0 \text {, }
$$

und jedes Element $f \in v_{n}(3)$ läßt sich als Summe eines Elementes von $\mu_{n}(3)$ und eines Elementes von $H \psi_{n-1}(3)$ darstellen:

$$
f=(f-H r f)+H r f
$$

mit

$$
r(f-H r f)=r f-r f=0,
$$

also

$$
(f-H r f) \in \mu_{n}(3) \text {. }
$$

Also ist $v_{n}(3)$ direkte Summe von $\mu_{n}(3)$ und $H \psi_{n-1}(3)$

$$
\nu_{n}(3)=\mu_{n}(3)+H \psi_{n-1}(3), \quad n \geqslant 2,
$$

oder vermöge des durch $P$ bewirkten Isomorphismus von $\nu_{n}(3)$ auf $\pi_{n}(Z)$ :

$$
\pi_{n}(Z)=P \pi_{n}(R)+P H \psi_{n-1}(3)
$$

wobei $P H$ die Gruppe $\psi_{n-1}(3)$ isomorph in $\pi_{n}(Z)$ abbildet, also auch

$$
\pi_{n}(Z) \approx P_{\pi_{n}}(R)+\psi_{n-1}(3) .
$$

Dies ist eine Verschärfung der Formel (7), (aber nur dann, wenn beide Summanden rechts von 0 verschieden sind). 
b) In folgendem Spezialfall läßt sich ein Homomorphismus mit den genannten Eigenschaften leicht angeben:

Das Element $\bar{A}$ der Zerlegung $3, \mathrm{~d}$.h. die Teilmenge $\bar{A}$ von $R$ sei in $R$ zusammenziehbar (dann gilt übrigens dasselbe für alle Elemente der Zerlegung, und wir wollen in diesem Falle 3 kurz eine zusammenziehbare Zerlegung nemnen). Dann ist übrigens $\psi_{n}(3)=\pi_{n}(\bar{A})$, und $\varphi_{n}(3)=0$, also $P \pi_{n}(R) \approx \pi_{n}(R)$.

Wir legen eine bestimmte Zusammenziehung von $\bar{A}$ in $R$ zugrunde, d. h. eine stetige Schar von Abbildungen $F_{t}(0 \leqslant t \leqslant 1)$ von $\bar{A}$ in $R$, bei welcher $F_{0}$ die identische Abbildung von $\bar{A}$ auf sich und $F_{1}$ die Abbildung von $\bar{A}$ auf einen Punkt von $R$ ist. Wir können dann in naheliegender Weise für jedes $f^{\prime} \epsilon \bar{A} \Sigma^{n-1}$ die Abbildungsschar $F_{t} f^{\prime}(0 \leqslant t \leqslant 1)$ als Element $f=H f^{\prime}$ von $R^{V^{n}}$ auffassen, derart, daß

$$
r H f^{\prime}=F_{0} f^{\prime}=f^{\prime}
$$

ist. Die Zuordnung $f=H f^{\prime}$ vermittelt dann die gesuchte homomorphe Abbildung von $\psi_{n-1}(3)=\pi_{n-1}(\bar{A})$ in $v_{n}(3)$. Nach Formel (9) gilt also

$$
\pi_{n}(Z)=P \pi_{n}(R)+P H \pi_{n-1}(\bar{A}),
$$

wobei $P$ und $P H$ Isomorphismen sind.

Satz $F$ : Unter den in Nr. 6a) genannten Voraussetzungen gilt für eine zusammenziehbare Zerlegung 3 von $R$ :

$$
\pi_{n}(Z) \approx \pi_{n}(R)+\pi_{n-1}(\bar{A}), \quad n \geqslant 2 .
$$

\section{Anwendungen}

a) Die in Nr. 2d) behandelten. Sphärenfaserungen

$$
S^{k q-1} / S^{q-1}=P_{k-1}\left(\Im_{q}\right)
$$

sind zusammenziehbare Zerlegungen; wir können also die Hurewiczschen Formeln in der verschärften Form (Satz F) anwenden:

Satz 6: Für die projektiven $\mathfrak{S}_{q}$-Räume $P_{k-1}\left(\mathfrak{S}_{q}\right)(q=1,2,4 ; k=$ $2,3, \ldots$ und $q=8 ; k=2$ ) gilt

$$
\pi_{n}\left(P_{k-1}\left(\Im_{q}\right)\right) \approx \pi_{n}\left(S^{k q-1}\right)+\pi_{n-1}\left(S^{\alpha-1}\right), n \geqslant 2 .
$$

Satz $6^{\prime}:$ Für $q=2,4,8$ gilt

$$
\pi_{n}\left(S^{q}\right) \approx \pi_{n}\left(S^{2 q-1}\right)+\pi_{n-1}\left(S^{q-1}\right), \quad n \geqslant 2 .
$$


Korallar zu Satz 6: 1. Für den reellen projektiven Raum $P_{k-1}\left(\Im_{1}\right)=$ $P^{k-1}$ gilt $\left.^{29}\right)$

$$
\pi_{n}\left(P^{k-1}\right) \approx \pi_{n}\left(S^{k-1}\right), \quad n \geqslant 2,
$$

was auch aus einem Satz von Hurewicz (s. ${ }^{8}$ ), Satz IV) folgt.

2. Für den komplexen projektiven Raum $P_{k-1}\left(\Im_{2}\right)=K_{k-1}$ gilt' $\left.^{30}\right)$

und

$$
\pi_{2}\left(K_{k-1}\right) \approx \pi_{1}\left(S^{1}\right) \approx(5
$$

$$
\pi_{n}\left(K_{k-1}\right) \approx \pi_{n}\left(S^{2 k-1}\right), \quad n \geqslant 3 .
$$

Bemerkung zu Satz $6^{\prime}$ : Es ist kein Fall bekannt, wo beide Summanden von 0 verschieden sind. Für $q=2$ erhält man den Hurewicz'schen Satz ${ }^{31}$ ), daß für $n \geqslant 3 \pi_{n}\left(S^{2}\right) \approx \pi_{n}\left(S^{3}\right)$ ist. Für $q=4$ und 8 entsteht die Darstellung von $\pi_{n}\left(S^{q}\right)$ als direkte Summe gemäß Formel (10) mit den dortigen Bezeichnungen folgendermaßen:

$$
\pi_{n}\left(S^{q}\right)=P \pi_{n}\left(S^{2 q-1}\right)+P H \pi_{n-1}\left(S^{q-1}\right),
$$

wobei $P \pi_{n}\left(S^{2 q-1}\right) \approx \pi_{n}\left(S^{2 q-1}\right)$ und $P H \pi_{n-1}\left(S^{q-1}\right) \approx \pi_{n-1}\left(S^{q-1}\right)$ ist $\left.{ }^{32}\right)$.

b) Die Anwendung der Hurewicz'schen Formeln (Satz E) auf die in Nr. 2e) beschriebenen Zerlegungen $3^{\prime}$ der Stiefel'schen Mannigfaltigkeiten $V_{n},{ }_{m}$

$$
V_{n, m} / V_{n-k, m-k}=V_{n, k}, \quad 0<k<m<n \text { (also } n \geqslant 3 \text { ) }
$$

ergibt (unter Verwendung der in Nr. $2 \mathrm{~d}$ und $6 \mathrm{~b}$ erklärten Bezeichnungen):

$$
\left.\begin{array}{rlrl}
\pi_{r}\left(V_{n, k}\right) / P \pi_{r}\left(V_{n, m}\right) & \approx \psi_{r-1}\left(V_{n, m} / V_{n-k, m-k}\right) & & \text { für } r \geqslant 2, \\
\pi_{1}\left(V_{n, k}\right) & =P \pi_{1}\left(V_{n, m}\right) & & . \\
P \pi_{r}\left(V_{n, m}\right) & \approx \pi_{r}\left(V_{n, m}\right) / \varphi_{r}\left(V_{n, m} / V_{n-k, m-k}\right) & & \text { für } r \geqslant 1, \\
\varphi_{r}\left(V_{n, m} / V_{n-k, m-k}\right) & \approx \pi_{r}\left(V_{n-k, m-k}\right) / \psi_{r}\left(V_{n, m} / V_{n-k, m-k}\right) & & \text { für } r \geqslant 1 .
\end{array}\right\}
$$

29) Es ist $\pi_{k}\left(S^{n}\right)=0$ für $k<n, \pi_{n}\left(S^{n}\right)$ die unendliche zyklische Gruppe, die von der Klasse der Identität der $S^{n}$ erzeugt wird, und $\pi_{n}\left(S^{1}\right)=0$ für $n \geq 2$. Vgl. s), S. 115 oben (diese Aussagen stützen sich auf bekannte Sätze, s. ${ }^{1}$ ), Kp. XIII).

30) Wir bezeichnen immer mit $\left(5\right.$ die additive Gruppe der ganzen Zahlen, mit $\mathfrak{G}_{2}$ die Restklassengruppe von $(5 \bmod .2$.

31) s. $\left.{ }^{8}\right)$, S. 119.

32) Wie man direkt einsehen kann, ist $P H$ hier nichts anderes als die Freudenthal'sche Einhängung (E (vgl, $\left.{ }^{38}\right)$ ); wir werden dies später (Nr. 10h und 13a) im Rahmen allgemeinerer Utberlegungen zeigen. 
(Die zweite dieser Formeln folgt aus $\left(7^{\prime}\right)$, weil $V_{n-k, m-k}$ zusammenhängend ist.)

Wir setzen in (11) $k=1$, also $m \geqslant 2 ; V_{n},{ }_{1}$ ist der $S^{n-1}$ homöomorph, also ist $\pi_{r}\left(V_{n, 1}\right)=0$ für $r \leqslant n-2$, also folgt:

oder

$$
\psi_{r-1}\left(V_{n, m} / V_{n-1, n-1}\right)=0 \quad \text { für } r \leqslant n-2,
$$

ferner

$$
\psi_{r}\left(V_{n, m} / V_{n-1, m-1}\right)=0 \quad \text { für } r \leqslant n-3 \text {, }
$$

also $\pi_{r}\left(V_{n, m}\right)=\psi_{r}\left(V_{n, m} / V_{n-1, m-1}\right) \approx \pi_{r}\left(V_{n-1, m-1}\right) \quad$ für $r \leqslant n-3$;

anders formuliert:

Satz 7: Für alle $n \geqslant r+2$ ist $\pi_{r}\left(V_{n, m}\right) \approx \pi_{r}\left(V_{n+1, m+1}\right)$.

c) Aus Satz 7 ergeben sich leicht einige Folgerungen.

Wenn $n-m+1 \geqslant r+2$ ist, so gilt

$$
\pi_{r}\left(V_{n, m}\right) \approx \pi_{r}\left(V_{n-1, m-1}\right) \approx \cdots \approx \pi_{r}\left(V_{n-m+1,1}\right)
$$

aber $V_{n-m+1,1}$ ist der $S^{n-m}$ homöomorph, und da wir $r \leqslant n-m-1$ voraussetzen, ist $\pi_{r}\left(V_{n-m+1,1}\right)=0$, und wir erhalten ein schon von Stiefe $\left.{ }^{33}\right)$ angegebenes Resultat:

Satz 8: Wenn $r \leqslant n-m-1$ ist, so ist $\pi_{r}\left(V_{n, m}\right)=0$.

Korollar: Wenn $n-m \geqslant 2$ ist, so ist $V_{n, m}$ einfach zusammenhängend.

Setzen wir in Satz $7 m=n-1$, so folgt für $n \geqslant r+2$ :

$$
\pi_{r}\left(V_{n+1, n}\right) \approx \pi_{r}\left(V_{n, n-1}\right) \approx \cdots \approx \pi_{r}\left(V_{r+2, r+1}\right) .
$$

$V_{n+1, n}$ ist zur Gruppe aller $(n+1)$-reihigen orthogonalen Matrizen mit der Determinante +1 , also zur Gruppe aller eigentlichen Drehungen $\operatorname{der} S^{n}$ homöomorph; wir bezeichnen diese Gruppe mit $\Gamma_{n}$. Dann können wir das letzte Resultat so formulieren:

Satz 9 : Für die Homotopiegruppen der Gruppe $\Gamma_{n}($ aller $(n+1)$-reihigen orthogonalen Matrizen mit der Determinante +1 ) gilt

für alle $n \geqslant r+1$.

$$
\pi_{r}\left(\Gamma_{n}\right) \approx \pi_{r}\left(\Gamma_{r+1}\right)
$$

32) s. ${ }^{14}$ ), S. 19, Satz 8 . 
Die $r$-te Homotopiegruppe von $\Gamma_{n}$ hat also für ,fast alle $n^{*}$, nämlich für alle $n \geqslant r+1$, dieselbe Struktur. Diese ist für $r=1$ und $r=2$ schon bekannt $\left.{ }^{34}\right)$ : es ist $\pi_{1}\left(I_{2}\right) \approx\left(5_{2}{ }^{30}\right)$ und $\pi_{2}\left(\Gamma_{3}\right)=0\left(\right.$ daß $\pi_{1}\left(\Gamma_{2}\right)$ zyklisch von der Ordnung 2 ist, folgt aus der Homöomorphie von $\Gamma_{2}$ zum reellen 3-dimensionalen projektiven Raum $P^{3}$; auf den Fall $r=2$ kommen wir in Nr. 13 zurück). Es sei hier erwähnt, daß ich auch für $r=3,4$ und 5 die Struktur von $\pi_{r}\left(\Gamma_{r+1}\right)$ habe bestimmen können $\left.{ }^{35}\right)$; es ist ${ }^{30}$ )

$$
\pi_{3}\left(\Gamma_{4}\right) \approx \mathfrak{b}, \pi_{4}\left(\Gamma_{5}\right)=0, \pi_{5}\left(\Gamma_{6}\right)=0 .
$$

d) Einen zu Satz 9 analogen Satz erhalten wir für die unitär-unimodulare Gruppe $A_{n}$, d. h. die Gruppe der unitären $(n+1)$-reihigen Matrizen mit der Determinante +1 , wenn wir von folgender Restklassenzerlegung (s. Nr. 2c) dieser Gruppe ausgehen:

$U$ sei die Gruppe derjenigen Matrizen $\left(u_{i k}\right)$ von $A_{n}(n \geqslant 2)$, für welche $u_{1 k}=\delta_{1 k}(k=1, \ldots, n+1)$ ist; sie ist mit $A_{n-1}$ isomorph. Zwei Matrizen von $A_{n}$ gehören in dieselbe (Links-) Restklasse nach $U$, wenn sie in der ersten Zeile übereinstimmen. Jede Restklasse ist also durch die $(n+1)$ komplexen Zablen $u_{1 k}$ mit $\sum_{k=1}^{n+1}\left|u_{1 k}\right|^{2}=1$ charakterisiert, d.h. der Zerlegungsraum dieser Restklassenzerlegung ist der $S^{2 n+1}$ homöomorph, und wir können sie nach Nr. 2 d kurz durch

$$
A_{n} / A_{n-1}=S^{2 n+1}
$$

beschreiben. Nach Nr. $2 \mathrm{e}$ ist es eine retrahierbare Zerlegung. $-S^{2 n+1}$ läßt sich also als Wirkungsraum von $A_{n}$ auffassen; das läßt sich auch so deuten: man kann jeder Matrix $a \in A_{n}$ eine unitäre Transformation $T_{a}$ in $(n+1)$ komplexen Variabeln, oder, wenn man $T_{a}$ in reelle Gleichungen aufspaltet, eine orthogonale Transformation in $(2 n+2)$ reellen Variabeln (eine Drehung der $S^{2 n+1}$ ) zuordnen.

Wir wenden den Satz $\mathbf{E}$ auf die Zerlegung $A_{n} / A_{n-1}=S^{2 n+1}(n \geqslant 2)$ an und erhalten:

$$
\begin{aligned}
& \pi_{r}\left(S^{2 n+1}\right) / P \pi_{r}\left(A_{n}\right) \approx \psi_{r-1}\left(A_{n} / A_{n-1}\right) \quad r \geqslant 2 \\
& \text { also } \quad \psi_{r-1}\left(A_{n} / A_{n-1}\right)=0 \text { für } r \leqslant 2 n \text {, } \\
& \text { oder } \quad \psi_{r}\left(A_{n} / A_{n-1}\right)=0 \quad \text { für } r \leqslant 2 n-1 \text {. } \\
& \text { Ferner } \quad P \pi_{r}\left(A_{n}\right)=0 \text { für } r \leqslant 2 n \text {, auch für } r=1 \text {, } \\
& \text { also } \quad \pi_{r}\left(A_{n}\right) \approx \pi_{r}\left(A_{n-1}\right) / \psi_{r}\left(A_{n} / A_{n-1}\right) \text { für } 1 \leqslant r \leqslant 2 n \\
& \text { und } \quad \pi_{r}\left(A_{n}\right) \approx \pi_{r}\left(A_{n-1}\right) \quad \text { für } 1 \leqslant r \leqslant 2 n-1 \text {. }
\end{aligned}
$$

34) Wegen $\pi_{1}\left(\Gamma_{n}\right)$ vgl. man: Stiefel $\left.{ }^{14}\right)$, S. 13, Nr. 4; wegen $\pi_{2}\left(\Gamma_{n}\right)$ Whitney (Bull. Am. Math. Soc. 43 [1937], S. 798).

35) Der Beweis wird demnächst veröffentlicht werden. 
Diese letzte Gleichung wenden wir wiederholt solange an, als noch $n \geqslant \frac{r+1}{2}$ ist und erhalten je nach der Parität von $r$ :

$$
\begin{aligned}
\pi_{2 s+1}\left(A_{n}\right) & \approx \pi_{2 s+1}\left(A_{n-1}\right) \approx \cdots \approx \pi_{2 s+1}\left(A_{s}\right) \\
\pi_{2 s}\left(A_{n}\right) & \approx \pi_{2 s}\left(A_{n-1}\right) \approx \cdots \approx \pi_{2 s}\left(A_{s}\right)
\end{aligned}
$$

und

$$
\pi_{1}\left(A_{n}\right) \approx \pi_{1}\left(A_{n-1}\right) \quad \approx \cdots \approx \pi_{1}\left(A_{1}\right) \text {. }
$$

Satz 10: Für $A_{n}$, die unitär-unimodulare Gruppe in $(n+1)$ Variabeln, gilt, wenn $n \geqslant s>0$ ist:

$$
\pi_{2 s+1}\left(A_{n}\right) \approx \pi_{2 s+1}\left(A_{s}\right) \quad \text { und } \quad \pi_{2 s}\left(A_{n}\right) \approx \pi_{2 s}\left(A_{s}\right),
$$

ferner für $n \geqslant 1: \pi_{1}\left(A_{n}\right) \approx \pi_{1}\left(A_{1}\right)$.

$A_{1}$ ist der multiplikativen Gruppe der Quaternionen vom Betrage $]$ isomorph, also der $S^{3}$ homöomorph; also ist ${ }^{30}$ )

$$
\pi_{1}\left(A_{1}\right)=0, \pi_{2}\left(A_{1}\right)=0, \pi_{3}\left(A_{1}\right) \approx \pi_{3}\left(S^{3}\right) \approx 5 .
$$

Korollar zu Salz 10: Für $n \geqslant 1$ ist $\pi_{1}\left(A_{n}\right)=0, \pi_{2}\left(A_{n}\right)=0, \pi_{3}\left(A_{n}\right) \approx(5$.

Auf $\pi_{4}\left(A_{n}\right)$ und $\pi_{5}\left(A_{n}\right)$ kommen wir in Nr. 16 zurück (es ist $\pi_{4}\left(A_{n}\right)=0$ und $\pi_{5}\left(A_{n}\right) \approx(5$ für $n \geqslant 2)$.

\section{\$ 4. Sätze über Schnittflächen und Schnittelemente}

\section{Notwendige Bedingungen für die Existenz einer Schnittfläche}

a) 3 sei eine retrahierbare Zerlegung des Kompaktums $R$; für alle Ausführungen dieses Paragraphes sollen dabei die Voraussetzungen und Bezeichnungen der Nummern $6 \mathrm{a}$ ) und $6 \mathrm{~b}$ ) gelten.

Unter einer Schnittfläche von 3 verstehen wir (vgl. Nr. 1g) eine Abbildung $j \in R^{Z}$, deren Spur $P j \in Z^{Z}$ die Identität von $Z$ ist.

Die natürliche Zerlegung des topologischen Produktes $Z \times \bar{A}$ (wo $Z$ und $\bar{A}$ Kompakten sind), deren Zerlegungsraum mit $Z$ homöomorph ist, besitzt immer eine Schnittfläche (vgl. Nr. 2a).

b) Wenn die Zerlegung 3 von $R$ eine Schnittfläche besitzt, so ist jede Abbildung $F \in Z S^{n}$ Spur einer Abljildung $f \in R s^{n}$, nämlich von $f=j F$ :

$$
P f=P j F=F .
$$


Nach dem Korollar zu Satz $\mathrm{E}$ ist also für $n \geqslant 2$

$$
\psi_{n-1}(3)=0 \text {. }
$$

(Als Punkt $a \in \bar{A}$ im Sinne von Nr. 6 a ist dabei $j(A)$ zu wählen.)

c) Ordnen wir der Abbildungsklasse von $F \in Z S^{n}$ die Abbildungsklasse von $j F \in R S^{n} \mathrm{zu}$, so ist dadurch eine homomorphe Abbildung von $\pi_{n}(Z)$ in $\pi_{n}(R)$ gegeben. Wir schreiben der Kürze halber auch für diese Abbildungsklassen $F$ und $j F$ (das ist zwar nicht ganz korrekt, weil nicht jede zur Abbildung $j F$ homotope Abbildung in der Form $j G, G \in Z S^{n}$, darstellbar zu sein braucht, und man somit zwischen $j\{F\}$ und $\{j F\}$ unterscheiden müßte; für das Rechnen in den Homotopiegruppen macht es aber nichts aus). Wegen

$$
P j F=F
$$

ist die genannte Zuordnung $j$ sogar ein Isomorphismus:

$$
\pi_{n}(Z) \approx j \pi_{n}(Z) \subset \pi_{n}(R) .
$$

Der Durchschnitt der Untergruppen $j \pi_{n}(Z)$ und $\varphi_{n}(3)$ von $\pi_{n}(R)$ ist die Null; denn aus $f \in \varphi_{n}(3)$ folgt $P f=0$, und hieraus, wenn $f \in j \pi_{n}(Z)$ ist, auch $f=0$ (weil $P$ die Gruppe $j \pi_{n}(Z)$ isomorph abbildet). Ferner läßt sich jedes Element $f$ von $\pi_{n}(R)$ als Summe eines Elementes von $\varphi_{n}(3)$ und eines Elementes von $j \pi_{n}(Z)$ darstellen:

$$
f=(f-j P f)+j P f,
$$

wobei $P(f-j P f)=P f-P f=0$, also $(f-j P f) \in \varphi_{n}(3)$ ist.

Also ist $\pi_{n}(R)$ direkte Summe von $j \pi_{n}(Z)$ und $\varphi_{n}(3)(n \geqslant 2)$ :

$$
\pi_{n}(R)=j \pi_{n}(Z)+\varphi_{n}(3) .
$$

Wegen $\psi_{n}(3)=0$ ist außerdem $\varphi_{n}(3) \approx \pi_{n}(\bar{A}) \quad(n \geqslant 1)$.

Satz G: Wenn die Zerlegung 3 von $R$ eine Schnittfläche besitzt, so gilt (vgl. Nr.6a und $6 b$ )

$$
\begin{array}{rlrl}
\text { I) } \psi_{n}(\mathcal{3}) & =0 & & n \geqslant 1 \\
\text { II) } \pi_{n}(R) \approx \pi_{n}(Z)+\pi_{n}(\bar{A}) & & n \geqslant 2 .
\end{array}
$$


Satz $G$ gilt insbesondere für das topologische Produkt $Z \times A$; das kann man auch leicht direkt (ohne Benützung einer Schnitttläche) einsehen ${ }^{36}$ ). Wichtig ist, daß in der Struktur der Homotopiegruppen eine Zerlegung 3 mit Schnittfläche sich nicht vom entsprechenden topologischen Produkt unterscheidet.

Für zusammenziehbare Zerlegungen $3(\mathrm{Nr} .7 \mathrm{~b})$ von $R$ können wir die Bedingung $\psi_{n}(3)=0(n \geqslant 1)$ weiter diskutieren. In diesem Falle ist nämlich

$$
\psi_{n}(3)=\pi_{n}(\bar{A}) \quad n \geqslant 1 .
$$

Somit gilt folgendes

Korollar zu Satz $G, I$ : Wenn die zusammenziehbare Zerlegung 3 von $R$ eine Schnittfläche besitzt, so ist $\pi_{n}(\bar{A})=0$ für alle $n \geqslant 1$.

Wenn $\bar{A}$ endlichdimensional ist, so bedeutet das Verschwinden sämtlicher Homotopiegruppen von $\bar{A}$, daß $\bar{A}$ (in sich) auf einen Punkt zusammenziehbar ist (Hurewicz ${ }^{37}$ ). Wir können also das Korollar auch so formulieren:

3 sei eine zusammenziehbare Zerlegung, ihre Elemente seien (endlichdimensional und) nicht in sich zusammenziehbar; dann besitzt 3 keine Schnittfläche.

So besitzt z. B. die Faserung einer Sphäre in geschlossene Mannigfaltigkeiten sicher keine Schnittfläche (was auch leicht aus Homologiebetrachtungen folgt).

10. Sphärische Zerlegungen $\left(Z=S^{m}\right)$.

a) Wir nennen eine Zerlegung von $R$ sphärisch und bezeichnen sie mit $\boldsymbol{Z}_{m}$, wenn ihr Zerlegungsraum $Z$ der $m$-dimensionalen Sphäre $S^{m}$ homöomorph ist $(m \geqslant 2)$.

Für eine solche Zerlegung $3_{m}$ von $R$ können wir leicht eine hinreichende Bedingung für die Existenz einer Schnittfläche angeben, nämlich

$$
\psi_{m-1}\left(3_{m}\right)=0
$$

daraus folgt nämlich nach dem Korollar zu Satz E (Nr. 6), daß jede Abbildung $f \in Z \mathrm{Ss}^{m}$ eine Spur ist. Wir können als Urbildsphäre für die $m$ te Homotopiegruppe auch $Z$ selbst (und als ausgezeichneten Punkt $x_{0}$

38) etwa so, wie in Seifert-Threlfall, Lehrbuch der Topologie, S. 156, in $\$ 43$ der dort als Beispiel 1 angeführte Satz bewiesen wird.

${ }^{37)}$ Proc. Akad. Amst. 38 (1935), S. 522, Satz IV, in Verbindung mit Satz II. 
(vgl. Nr. 6a) den Punkt $A \in Z$ ) wählen; ist $\psi_{m-1}\left(3_{m}\right)=0$, so muß also die Identität von $Z$ eine Spur sein. Also gilt mit Rücksicht auf Satz G, I:

Satz 11: In einer sphärischen Zerlegung $3_{m}$ von $R$ gibt es dann und nur dann eine Schnittfläche, wenn $\psi_{m-1}\left(3_{m}\right)=0$ ist.

b) $\mathfrak{Z}_{m}$ sei eine sphärische Zerlegung von $R$, und $Z$ ihr Zerlegungsraum. Wir verwenden im folgenden dieselben Bezeichnungen wie in Nr. 6d, und fassen wie dort die Gruppe $\pi_{n}(Z)$ als Gruppe der Komponenten von $Z \nabla^{n}$ auf (wo $Z V^{n}$ den Raum derjenigen Abbildungen $F$ von $V^{n}$ in $Z$ bedeutet, für welche $F\left(\sum^{n-1}\right)=A \in Z$ ist).

$T$ sei eine stetige Abbildung von $V^{m}$ auf $Z$, bei welcher das Innere von $V^{m}$ topologiseh auf $Z-A$ und $\Sigma^{m-1}$ auf $A$ abgebildet wird. (Die Klasse von $T$ in $Z^{m}$ ist eines der beiden erzeugenden Elemente von $\pi_{m}(Z)=$ $\pi_{m}\left(S^{m}\right) \approx\left(\mathfrak{G}\right.$.) Es gibt immer eine Abbildung $t \in R^{\nabla^{m}}$, derart, daß $P t=T$ ist; $t$ ist im Innern von $V^{m}$ topologisch. ( $t T^{-1}$ ist eine Abbildung von $Z$ in $R$, deren Spur die Identität von $Z$ ist; sie ist aber im allgemeinen in $A$ nicht eindeutig!)

Wir nennen $t$ ein Schnittelement der Zerlegung $3_{m}$; sein „Rand" $r t=$ $t^{\prime} \in \bar{A} \Sigma^{m-1}$ wird sich als besonders wichtig für die Struktur der Zerlegung erweisen (Bedeutung von $r$ s. Nr. $6 \mathrm{~d}$ ).

Die Abbildungsklasse von $t$, aufgefaßt als Element von $v_{m}\left(3_{m}\right)$ ist, ebenso wie die Klasse von $T$, bis auf das Vorzeichen eindeutig bestimmt. Wir setzen noch fest, daß $t$ (bei fester Orientierung von $V^{m}$ und $Z$, die auch im folgenden immer beibehalten werde) den Abbildungsgrad +1 haben soll; dann ist die Klasse von t bestimmt.

Die folgenden Überlegungen gestatten nun, bei sphärischen Zerlegungen $\mathfrak{Z}_{m}$ mit Hilfe eines Schnittelementes $t$ die Gruppen $\psi_{n-1}\left(3_{m}\right)$ wenigstens für gewisse Dimensionszahlen $n$ näher zu beschreiben, also diejenigen Abbildungen von $\sum^{n-1}$ in $\bar{A}$ anzugeben, welche in $R$ zusammenziehbar sind; Abbildungen, die aus einer Abbildung von $\sum^{n-1}$ auf $\sum^{m-1}$ und der Abbildung $t^{\prime}$ von $\sum^{m-1}$ in $\bar{A}$ zusammengesetzt sind, haben sicher diese Eigenschaft, für gewisse $n$ sind es - wie wir sehen werden - die einzigen.

c) Wir definieren nun für jede Abbildung $h$ von $\Sigma^{n-1}$ in $\Sigma^{m-1}$ eine Abbildung $E h$ von $V^{n}$ in $V^{m}$ vermöge folgender Zuordnung, die ,Einhängung" heißen soll; $\mathfrak{x}$ (bzw. y) sei ein Vektor des $R^{n}\left(R^{m}\right)$, in welchem $V^{m}\left(V^{n}\right)$ durch $\mathfrak{x}^{2} \leqslant 1\left(\mathfrak{y}^{2} \leqslant 1\right)$ dargestellt wird; $h$ sei gegeben durch die für $\mathfrak{x}^{2}=1$ definierte Vektorfunktion

$$
\mathfrak{y}=h(\mathfrak{x}) \quad \text { mit } \quad(h(\mathfrak{x}))^{2}=1 .
$$


Dann wird $E h$ definiert durch

$$
\begin{array}{lll}
\mathfrak{y}=|\mathfrak{x}| h\left(\frac{\mathfrak{x}}{|\mathfrak{x}|}\right) & \text { für } & 0<|\mathfrak{x}| \leqslant 1 \\
\mathfrak{y}=0 & \text { für } & \mathfrak{x}=0 .
\end{array}
$$

$F=T(E h)$ ist eine Abbildung von $V^{n}$ in $Z$, für welche $F\left(\sum^{n-1}\right)=A$ ist, d. h. $F \in Z^{V^{n}}$; sie bestimmt also ein Element $T(E h)$ der Gruppe $\pi_{n}(Z)$ oder $\pi_{n}\left(S^{m}\right)$. Verstehen wir unter $\mathfrak{E}$ die Einhängung im Sinne von Freudentha $\left.7^{38}\right)$, und unter $h$ jetzt das durch die Abbildung $h$ von $\sum^{n-1}$ in $\sum^{m-1}$ bestimmte Element von $\pi_{n-1}\left(S^{m-1}\right)$, so gilt für das Element $T(E h)$ von $\pi_{n}\left(S^{m}\right)$ entweder $T(E h)=+$ \&h oder $T(E h)=-\mathbb{E} h$; bei der am Schluß von Nr. 10b gemachten Festsetzung über $T$ ist

$$
T(E h)=\mathfrak{E} h .
$$

d) $f=t(E h)$ ist eine Abbildung von $V^{n}$ in $R$ mit $f\left(\sum^{n-1}\right) \subset \overline{A \text {, also }}$ eine Abbildung aus $R V^{n}$. Setzen wir $t^{\prime}=r t$, so ist

$$
r(t(E h))=(r t) h=t^{\prime} h .
$$

$f=t(E h)$ bestimmt eine Klasse $t(E h) \in \nu_{n}\left(3_{m}\right)$; für diese gilt wegen $P t=T$

$$
P(t(E h))=P t(E h)=\mathbb{E} h .
$$

Wir ordnen nun jedem Element von $\pi_{n-1}\left(S^{m-1}\right)$, repräsentiert durch eine Klasse $h$ von Abbildungen der Sphäre $\sum^{n-1}$ in $\sum^{m-1}$, diejenige Klasse von Abbildungen der $\Sigma^{n-1}$ in $\bar{A} \mathrm{zu}$, in welcher $t^{\prime} h$ enthalten ist, und nennen diese Klasse, aufgefaßt als Element von $\pi_{n-1}(\bar{A})$, auch $t^{\prime} h$. Da für 2 Abbildungen $h_{1}, h_{2}$ von $\sum^{n-1}$ in $\sum^{m-1}$

$$
t^{\prime}\left(h_{1}+h_{2}\right)=t^{\prime} h_{1}+t^{\prime} h_{2}
$$

ist, bedeutet diese durch $t^{\prime}=r t$ vermittelte Zuordnung eine homomorphe Abbildung von $\pi_{n-1}\left(S^{m-1}\right)$ in $\pi_{n-1}(\bar{A})$ :

$$
\pi_{n-1}\left(S^{m-1}\right) \sim t^{\prime} \pi_{n-1}\left(S^{m-1}\right) \subset \pi_{n-1}(\bar{A}) .
$$

Zunächst folgt nun aus (13):

$$
t^{\prime} \pi_{n-1}\left(S^{m-1}\right) \subset \psi_{n-1}\left(3_{m}\right) .
$$

38) Definition der Einhängung s. ${ }^{23}$ ), S. 303. 
In der Tat: wenn $f^{\prime} \in \pi_{n-1}(\bar{A})$ von der Form $t^{\prime} h$ ist $\left(h \epsilon \pi_{n-1}\left(S^{m-1}\right)\right)$, so gibt es ein Element $f \epsilon v_{n}\left(3_{m}\right)$, derart, daß $f^{\prime}=r f$ ist, nämlich $f=t(E h)$. - Aus (14) folgt, daß für dieses $f$ gilt: $P f=\mathbb{E} h$. Nun entspricht aber (Nr. 6d) jedem Element $F \in \pi_{n}(Z)$ ein wohlbestimmtes Element $f \in \nu_{n}\left(3_{m}\right)$, derart, daß $P f=F$ ist, und wenn wir $r f=f^{\prime}$ setzen, so ist die Zuordnung

$$
F \rightarrow f^{\prime}
$$

eine homomorphe Abbildung von $\pi_{n}(Z)$ auf $\psi_{n-1}\left(\boldsymbol{Z}_{m}\right)$. Wenn nun $F=\mathfrak{E} h$ ist, so muß nach (14) das zugehörige $f \epsilon v_{n}\left(3_{m}\right)$ von der Form $f=t(E h)$ sein, dann ist also $f^{\prime}=t^{\prime} h$. Das gilt für jedes Element $h$ von $\pi_{n-1}\left(S^{m-1}\right)$.

Insbesondere gilt also folgender

Hilfssatz: $3_{m}$ sei eine sphärische Zerlegung, $t$ ein Schnittelement von $3_{m}$ und $t^{\prime}=r t$. Es gibt eine homomorphe Abbildung von $\pi_{n}(Z)$ auf $\psi_{n-1}\left(3_{m}\right)$ (es ist die oben erklärte Zuordnung $\left.F \rightarrow f^{\prime}\right)$, bei welcher die Untergruppe $\left[\tilde{E}_{n-1}\left(S^{m-1}\right)\right.$ von $\pi_{n}(Z)$ auf die Untergruppe $t^{\prime} \pi_{n-1}\left(S^{m-1}\right)$ von $\psi_{n-1}\left(3_{m}\right)$ abgebildet wird.

e) Gemäß der Freudenthal'schen Theorie der Abbildungen von Sphären auf Sphären läßt sich ${ }^{30}$ ) für alle $n<2 m-1$ (und bei ungeradem $m$ auch für $n=2 m-1$ ) jedes Element von $\pi_{n}\left(\mathcal{S}^{m}\right)$ bzw. von $\pi_{n}(Z)$ durch Einhängung erzeugen; es ist also für diese $n$

$$
\pi_{n}(Z)=\left[\boldsymbol{E}_{n-1}\left(S^{m-1}\right),\right.
$$

und aus dem Hilfssatz folgt dann:

Satz 12: $3_{m}$ sei eine sphärische Zerlegung, $t$ ein Schnittelement von $3_{m}$ (Definition s. Nr. 11b) und $t^{\prime}=r t$. Dann ist für $n<2 m-1$ (und bei ungeradem $m$ auch für $n=2 m-1$ )

$$
\psi_{n-1}\left(3_{m}\right)=t^{\prime} \pi_{n-1}\left(S^{m-1}\right)
$$

Insbesondere ist also $\psi_{m-1}\left(\mathfrak{Z}_{m}\right)=t^{\prime} \pi_{m-1}\left(S^{m-1}\right)$, d. h. $\psi_{m-1}\left(\mathfrak{Z}_{m}\right)$ ist die von der Klasse von $t^{\prime}$ erzeugte Untergruppe von $\pi_{m-1}(\bar{A})$.

Beweis der letzten Behauptung: Bezeichnen wir mit $e$ die Klasse der Identität der $S^{m-1}$, so ist $t^{\prime} e$ die Klasse von $t^{\prime}$ in $\pi_{m-1}(\bar{A})$, ferner für das Element $h \in \pi_{m-1}\left(S^{m-1}\right)$ mit dem Abbildungsgrad $g$

also

$$
h=g \cdot e
$$

$$
t^{\prime} h=t^{\prime}(g \cdot e)=g \cdot t^{\prime} e .
$$

39) ธ. $\left.{ }^{23}\right)$, S. 300 , Satz I. 
$t^{\prime} \pi_{m-1}\left(S^{m-1}\right)$ ist also genau die Untergruppe aller Vielfachen der Klasse von $t^{\prime}$ in $\pi_{m-1}(\bar{A})$.

Korollar zu Satz 12: In der zusammenziehbaren (s. Nr. $7 \mathrm{~b}$ ) sphärischen Zerlegung $3_{m}$ mit dem Schnittelement $t$ und $t^{\prime}=r t$ gilt für $n<2 m-1$ (und $n=2 m-1$ bei ungeradem $m$ )

$$
\pi_{n-1}(\bar{A})=t^{\prime} \pi_{n-1}\left(S^{m-1}\right) \text {. }
$$

f) Satz 13: $3_{m}$ sei eine sphärische Zerlegung von $R, t$ ein Schnittelement von $3_{m}$. Wenn der durch $t^{\prime}(=r t)$ bewirkte Homomorphismus von $\pi_{n-1}\left(S^{m-1}\right)$ in $\pi_{n-1}(\bar{A})$ ein Isomorphismus auf $\psi_{n-1}\left(3_{m}\right)$ ist, dann zerfällt $\pi_{n}(Z)$ in die direkte Summe

$$
\pi_{n}(Z)=P \pi_{n}(R)+\left[\mathbb{E} \pi_{n-1}\left(S^{m-1}\right),\right.
$$

und die Einhängung $\mathbb{E}$ bildet $\pi_{n-1}\left(S^{m-1}\right)$ isomorph ab; also

$$
\pi_{n}(Z) \approx P \pi_{n}(R)+\pi_{n-1}\left(S^{m-1}\right) .
$$

Beweis: Wenn die Voraussetzung erfüllt ist, so entspricht jêdem Element $f^{\prime}=t^{\prime} h \in \psi_{n-1}\left(3_{m}\right)$ ein bestimmtes Element $h \epsilon \pi_{n-1}\left(S^{m-1}\right)$. Die Zuordnung $f^{\prime} \rightarrow H f^{\prime}=t(E h)$ ist dann ein Homomorphismus von $\psi_{n-1}\left(3_{m}\right)$ in $v_{n}\left(3_{m}\right)$ mit den in Nr. 7 a geforderten Eigenschaften; nach Formel (9) gilt also

$$
\begin{aligned}
\pi_{n}(Z) & =P \pi_{n}(R)+P t\left(E \pi_{n-1}\left(S^{m-1}\right)\right) \\
& =P \pi_{n}(R)+E \pi_{n-1}\left(S^{m-1}\right) .
\end{aligned}
$$

Da $P H$ dabei die Gruppe $\psi_{n-1}\left(3_{m}\right)$ isomorph in $\pi_{n}(Z)$ abbildet, ist

$$
\text { PH } \psi_{n-1}\left(3_{m}\right)=\mathfrak{E}_{\pi_{n-1}}\left(S^{m-1}\right) \approx \psi_{n-1}\left(3_{m}\right),
$$

ferner nach Voraussetzung

$$
\psi_{n-1}\left(3_{m}\right)=t^{\prime} \pi_{n-1}\left(S^{m-1}\right) \approx \pi_{n-1}\left(S^{m-1}\right),
$$

also

$$
\left[\mathfrak{E} \pi_{n-1}\left(S^{m-1}\right) \approx \pi_{n-1}\left(S^{m-1}\right),\right.
$$

und dieser Isomorphismus wird offenbar gerade durch die Einhängung $\mathfrak{E}$ vermittelt.

Korollar zu Satz 13: Für $n<2 m-1$ (und bei ungeradem $m$ auch für $n=2 m-1$ ) gilt: Wenn die durch $t^{\prime}$ bewirkte Abbildung von $\pi_{n-1}\left(S^{m-1}\right)$ $\mathrm{n} \pi_{n-1}(\bar{A})$ isomorph ist, so ist $P_{\pi_{n}}(R)=0$. 
Beweis: Für diese $n$ ist nach Satz $12 \psi_{n-1}\left(3_{m}\right)=t^{\prime} \pi_{n-1}\left(S^{m-1}\right)$, und außerdem $\mathbb{E} \pi_{n-1}\left(S^{m-1}\right)=\pi_{n}\left(S^{m}\right)$ (was im Beweis von Satz 12 benützt wird).

g) Wir betrachten nun noch den speziellen Fall einer sphärischen Zerlegung $\mathcal{Z}_{m}$ von $R$, bei welcher $\bar{A}$ der Sphäre $S^{m-1}$ homöomorph ist. $t$ sei ein Schnittelement von $3_{m}$; die Klasse der Abbildung $t^{\prime}=r t$ von $\Sigma^{m-1}$ in $\bar{A}$ läßt sich durch den Abbildungsgrad $k$ charakterisieren, der bei geeigneter Orientierung von $\bar{A}$ nicht-negativ ist. Wir bezeichnen eine Abbildung einer $(m-1)$-dimensionalen Sphäre in eine andere vom Grade $k$ (in unserem Fall der $\Sigma^{m-1}$ in $\bar{A}$ ) immer mit $T_{k}$ oder ausführlicher mit $T_{k}^{m-1}$.

Die Klasse von $t \in R^{V^{m}}$ (also auch die von $t^{\prime}=r t \in \bar{A} \Sigma^{m-1}$ ) ist nach Nr. 10c durch die Zerlegung $3_{m}$ eindeutig bestimmt. $\mathrm{Zu} 3_{m}$ gehört also eine nicht-negative ganze Zahl $k$, die die Eigenschaft hat: für jedes Schnittelement $t$ von $3_{m}$ ist $r t=T_{k}^{m-1}$. Wir nennen $k$ die charakteristische Zahl der Zerlegung $3_{m}$.

Aus den Sätzen dieser Nummer folgt für den betrachteten Spezialfall:

Satz 14: $3_{m}$ sei eine sphärische Zerlegung von $R$, bei welcher $\bar{A}$ der $S^{m-1}$ homöomorph ist, und $k$ sei ihre charakteristische Zahl. Dann gilt

a) Für $n<2 m-1$ (und bei ungeradem $m$ auch $n=2 m-1$ ) ist

$$
\psi_{n-1}\left(\mathbf{3}_{m}\right)=T_{k}^{m-1} \pi_{n-1}\left(S^{m-1}\right) .
$$

b) Wenn $k \neq 0$ ist, dann ist $P \pi_{m}(R)=0$.

Beweis: a) folgt direkt aus Satz 12, b) aus dem Korollar zu Satz 13, wenn man bemerkt, daß für $k \neq 0$ die durch $t^{\prime}=T_{k}$ bewirkte Abbildung von $\pi_{m-1}\left(S^{m-1}\right)$ in $\pi_{m-1}(\bar{A})$ isomorph ist: $T_{l}$ sei eine Abbildung vom Grade $l$ von $\Sigma^{m-1}$ in sich; wegen $T_{k} T_{l}=T_{k l} \in \bar{A} \Sigma^{m-1}$ folgt aus $T_{k} T_{l}=0$ auch $k \cdot l=0$, also $l=0$.

Für $n=m$ folgt aus Satz 14a) leicht (mit denselben Voraussetzungen und Bezeichnungen):

Korollar : Eine Abbildung von $\bar{A}$ in sich ist in $R^{\bar{A}}$ dann und nur dann zusammenziehbar, wenn ihr Grad ein Vielfaches von $k$ ist.

h) Insbesondere ist dann und nur dann die Zerlegung, von der in Satz 14 die Rede ist, zusammenziehbar (d. h. $\bar{A}$ in $R$ zusammenziehbar), wenn $k=1$ ist. In diesem Falle sind für alle $n \geqslant 2$ die Voraussetzungen von Satz 13 erfüllt; daraus folgt: 
Satz 15: In einer zusammenziehbaren sphärischen Zerlegung $\mathbf{3}_{m}$, in welcher $\bar{A}$ der $S^{m-1}$ homöomorph ist, gilt für alle $n \geqslant 2$

$$
\pi_{n}(Z)=P \pi_{n}(R)+\mathcal{E} \pi_{n-1}\left(S^{m-1}\right),
$$

wobei $P$ und $\mathbb{E}$ Isomorphismen von $\pi_{n}(R)$ bzw. $\pi_{n-1}\left(S^{m-1}\right)$ in $\pi_{n}(Z)$ sind.

Dieser Satz geht nur insofern über die Formel (10) und den Satz F (Nr. 7 b) hinaus, als er zeigt, daß unter der Voraussetzung $Z=S^{m}$ und $\bar{A}=S^{m-1}$ die a. a. O. $P H$ genannte Abbildung von $\pi_{n-1}(\bar{A})$ in $\pi_{n}(Z)$ mit der Freudenthal'schen „Einhängung“ identisch ist (vgl. auch Nr. 8a); für den Beweis dieser Tatsache allein wären natürlich die meisten Sätze dieser Nummer in ihrer Allgemeinheit entbehrlich gewesen.

\section{Gruppenzerlegungen}

a) 3 sei die Zerlegung einer kompakten topologischen Gruppe $G$ in Restklassen nach einer abgeschlossenen Untergruppe $U$; der Zerlegungsraum $Z$ ist ein Wirkungsraum von $G$ (vgl. Nr. $2 \mathrm{c}$ ). 3 sei retrahierbar; das ist sicher der Fall, wenn $G$ eine Lie'sche Gruppe ist (Nr. 2c). Als ausgezeichneten Punkt im Sinne von Nr. 6a wählen wir die Gruppeneins $e$ von $G$ (also $A=P e$, und $\bar{A}=U$ ). Ist $j$ eine Schnittfläche von 3 , so können wir immer annehmen, $\operatorname{daß} j(A)=e$ ist.

b) Wenn diese Zerlegung 3 von $G$ eine Schnittfläche $j$ besitzt, so ist durch

$$
f(u, B)=u \cdot j(B) \quad u \in U, B \in Z
$$

(wo - die Multiplikation in der Gruppe $G$ bedeutet) eine topologische Abbildung des topologischen Produkts $U \times Z$ auf $G$ gegeben, bei welcher $f(U, B)=\bar{B}$ ist; dann ist also $G$ dem topologischen Produkt $U \times Z$ homöomorph, und 3 ist der Links-Zerlegung von $U \times Z$ homöomorph (Nr. 1b) (d. h. der natürlichen Zerlegung von $U \times Z$, deren Zerlegungsraum $Z$ ist). Also

c) Satz 16:Ist W ein Wirkungsraum der geschlossenen Lie'schen Gruppe $G$ mit der Isotropiegruppe $U$, und besitzt die Zerlegung 3 von $G$ in Restklassen nach $U$ eine Schnittfläche, so ist $G$ dem topologischen Produkt $U \times W$ homöomorph, und 3 ist der Links-Zerlegung von $U \times W$ homöomorph. 
Hieraus und aus Satz 11 folgt das

Korollar: Die Sphäre $S^{m}$ sei ein Wirkungsraum der geschlossenen Lieschen Gruppe $G$, und $3_{m}$ die zugehörige (sphärische) Zerlegung von $G$ in Restklassen nach der Isotropiegruppe $U$. Dann gilt:

Wenn $\psi_{m-1}(3)=0$ ist, so ist $G$ dem topologischen Produkt $U \times S^{m}$ homöomorph.

\section{§. Anwendungen, insbesondere auf Sphären}

\section{Vorbereitendes über Sphärenabbildungen}

a) Wir benützen in diesem Paragraphen folgende bekannten Resultate aus der Theorie der Abbildungen von Sphären auf Sphären, die wir als Aussagen über die $n$ te Homotopiegruppe $\pi_{n}\left(S^{m}\right)$ der $m$-dimensionalen Sphäre $S^{m}$ formulieren (vgl. auch Anm. ${ }^{29}$ ) ${ }^{30}$ ):

$$
\begin{aligned}
\pi_{n}\left(S^{1}\right) & =0 & & \text { für } n \geqslant 2 \\
\pi_{n}\left(S^{m}\right) & =0 & & \text { für } n \leqslant m-1 \\
\pi_{m}\left(S^{m}\right) & \approx \tilde{5} & & \text { für } m \geqslant 1 \\
\pi_{m+1}\left(S^{m}\right) & \approx \mathfrak{G}_{2} & & \text { für } \left.m \geqslant 3{ }^{40}\right) \\
\pi_{m+2}\left(S^{m}\right) & =0 & & \text { für } \left.m \geqslant 3^{41}\right) .
\end{aligned}
$$

b) Mit $T_{k}^{m}$ (oder $T_{k}$ ) bezeichnen wir eine Abbildung der Sphäre $S^{m}$ in sich vom Grade $k$. Ist $f$ eine Abbildung der $S^{n}$ in die $S^{m}$, so ist auch $T_{k}^{m} f$ eine solche, und wir bezeichnen auch ihre Abbildungsklassen mit $f$ und $T_{k} f$ (statt $|f|$ und $\left\{T_{k}^{m} f\right\}$ ). Ordnet man dem Element $f \in \pi_{n}\left(S^{m}\right)$ das Element $T_{k}^{m} f \mathrm{zu}$, so entsteht eine homomorphe Abbildung von $\pi_{n}\left(S^{m}\right)$ in sich. - $\mathfrak{E}$ bedeute die Freudenthal'sche Einhängung $\left.{ }^{38}\right)$.

Hilfssatz $\left.{ }^{42}\right)$ : Für $m \geqslant 3$ ist $T_{2}^{m} \pi_{m+1}\left(S^{m}\right)=0$. - Oder: Für jede Abbildung $f$ der $S^{m+1}$ in die $S^{m}(m \geqslant 3)$ ist $T_{2} f$ zusammenziehbar.

Beweis: Es sei $m \geqslant 3$ und $f \in \pi_{m+1}\left(S^{m}\right)$; nach einem Satze von Freudenthal ${ }^{39}$ ) gibt es in der Klasse $f$ eine ,eingehängte" Abbildung:

$$
f==\mathfrak{E} g .
$$

40) s. Freudenthal ${ }^{23}$ ), S. 301. Ferner: L. Pontrjagin (C. R. Acad. Sc. de l'U.R.S.S. [1938] XIX, 147-149).

41) L. Pontrjagin (C. R. Acad. Sc. de l'U.R.S.S. [1938] XIX, 361-363).

42) Dieser Satz und ebenso das Korollar zu Satz 17 ergeben sich leicht aus allgemeinern Betrachtungen über den Homomorphismus $T_{k}^{m}$ von $\pi_{n}\left(S^{m}\right)$ in sich, auf welche wir an anderer Stelle eingehen werden. Wir ziehen es vor, hier einen kurzen direkten Beweis für den Hilfssatz anzugeben. 
Nun kann man aber leicht verifizieren, daß für beliebiges $n, m$ und $k$ und $g \in \pi_{n-1}\left(S^{m-1}\right)$

$$
T_{k}^{m} \mathfrak{E} g=\mathbb{E} T_{k}^{m-1} g
$$

ist. Die Abbildungsklasse $T_{2}^{m} f$ geht also durch (evtl. wiederholte) Einhängung aus $T_{2} h$ hervor, wo $h$ ein Element von $\pi_{3}\left(S^{2}\right)$ ist. Für die Hopfsche Invariante $\left.{ }^{43}\right) \gamma$ von $T_{2}^{2} h$ gilt:

$$
\gamma\left(T_{2}^{2} h\right)=4 \gamma(h)=\gamma(4 h)
$$

Die Elemente von $\pi_{3}\left(S^{2}\right)$ sind aber durch die Invariante $\gamma$ charakterisiert; denn $\gamma$ ist ein ganzzahliger, nichttrivialer Charakter von $\pi_{3}\left(S^{2}\right)$, und nach Satz $6^{\prime}$ ist $\pi_{3}\left(S^{2}\right) \approx \pi_{3}\left(S^{3}\right)$ eine unendliche zyklische Gruppe. Also folgt

$$
T_{2}^{2} h=4 h,
$$

also

$$
\mathfrak{E} T_{2}^{2} h=\mathfrak{E}(4 h)=4 \cdot \mathfrak{E} h=0,
$$

weil $\pi_{4}\left(S^{3}\right) \approx \mathfrak{G}_{2}$ ist. Also ist auch

$$
T_{2}^{m} f=\mathfrak{E} \mathfrak{E} \cdots \mathfrak{E} T_{2}^{2} h=0, \quad \text { q.e.d. }
$$

\section{Sphärenfaserungen}

a) Die in $\mathrm{Nr} 2 \mathrm{~d}$ beschriebenen Sphärenfaserungen

$$
S^{2 q-1} / S^{q-1}=S^{q}, \quad q=2,4,8
$$

sind zusammenziehbare sphärische Zerlegungen $\boldsymbol{Z}_{q}$, deren Elemente $(q-1)$-dimensionale Sphären sind. Nach Satz 15 (Nr 10h) gilt also für $n \geqslant 2$

$$
\pi_{n}\left(S^{q}\right)=P \pi_{n}\left(S^{2 q-1}\right)+\mathfrak{E} \pi_{n-1}\left(S^{q-1}\right),
$$

wobei $P$ die Gruppe $\pi_{n}\left(S^{2 q-1}\right)$ und $\mathbb{E}$ die Gruppe $\pi_{n-1}\left(S^{q-1}\right)$ isomorph in $\pi_{n}\left(S^{q}\right)$ abbildet Das ist genau der Satz $6^{\prime}(\mathrm{Nr} 8 \mathrm{a})$, nur dahin präzisiert, daß die dort $P H$ genannte Abbildung von $\pi_{n-1}\left(S^{q-1}\right)$ in $\pi_{n}\left(S^{q}\right)$ mit der Einhängung identisch ist. Wesentliche Abbildungen (für Abbildungen in Sphären ist ,,wesentlich" und ,nicht-zusammenziehbar" gleichbedeutend) der $S^{n}$ auf $S^{q}$ entstehen also auf 2 Arten: einerseits durch Projektion (bezüglicb der Zerlegung $3_{q}$ ) wesentlicher Abbildungen der $S^{n}$ auf $S^{2 q-1}$, andererseits durch Einhängung wesentlicher Abbildungen der $S^{n-1}$ auf $S^{q-1}$.

43) H. Hopf, Ưber die Abbildungen der 3-dimensionalen Sphäre auf die Kugelfläche (Math. Ann. 104 [1931], 637-655), vgl. auch ${ }^{12}$ ). Beweis, daß $\gamma$ ein Charakter von $\pi_{3}\left(S^{2}\right)$ ist: Freudenthal $\left.{ }^{23}\right)$, S. 305 . 
b) Wir heben noch den Teil dieses Resultates, der die Einhängung betrifft und der ein interessantes Gegenstück zu Sätzen von Freudenthal ${ }^{44}$ ) ist, besonders hervor (für $q=2$ trivial):

Satz 17: Für $q=4$ und 8 , und für beliebiges $n \geqslant 2$ bildet die Einhängung (E) die Gruppe $\pi_{n-1}\left(S^{q-1}\right)$ isomorph in die Gruppe $\pi_{n}\left(S^{q}\right)$ ab (d. h. aus $f \in \pi_{n-1}\left(S^{q-1}\right), \mathbb{E} f=0$ folgt $\left.f=0\right)$.

Nach Freudenthal ${ }^{45}$ ) gilt immer

$$
\mathfrak{E}\left(f+T_{-1} f\right)=0
$$

also folgt aus Satz 17 das

Korollar $\left.^{42}\right)$ : Für $m=3$ und 7 , und beliebiges $n$, und $f \in \pi_{n}\left(S^{m}\right)$ gilt

$$
T_{-1} f=-f \text {. }
$$

(Dagegen ist z. B. für $n=3$ und $m=2$ immer $T_{-1} f=f$.)

\section{4. k-Felder auf Sphären}

a) Wir bezeichnen mit $L_{m, k}$ die (in natürlicher Weise topologisierte) Mannigfaltigkeit aller an die $m$-dimensionale Sphäre $S^{m}$ tangentialen $k$-Systeme des $R^{m+1}$ (s. Nr. 2e), $0<k<m$. Fassen wir in jedem Punkt der $S^{m}$ die dort angreifenden tangentialen $k$-Systeme zu einer Teilmenge von $L_{m, k}$ zusammen, so ist diese Teilmenge eine Mannigfaltigkeit $V_{m, k}$ (Nr. $2 \mathrm{~g}$ ), und alle solchen Teilmengen bilden eine Zerlegung (sogar eine ,,reguläre Faserung“) von $L_{m, k}$; ihr Zerlegungsraum ist der $S^{m}$ homöomorph. Wir nennen sie die natürliche Zerlegung von $L_{m, k}$ und beschreiben sie kurz durch

$$
L_{m, k} / V_{m, k}=S^{m} .
$$

Eine Schnittfläche der natürlichen Zerlegung von $L_{m, k}$ heißt auch ${ }^{46}$ ) ein $k$-Feld auf $S^{m}$; man kann ein $k$-Feld auf $S^{m}$ auffassen als System von $k$ tangentialen Vektorfeldern auf $S^{m}$, die in jedem Punkt der $S^{m}$ paarweise orthogonal sind. Ein 1-Feld ist dasselbe wie ein tangentiales Vektorfeld; $L_{m, 1}$ ist der Raum der gerichteten Linienelemente der $S^{m}$.

b) $L_{m, k}$ ist der Mannigfaltigkeit $V_{m+1, k+1}$ homöomorph, und die natürliche Zerlegung von $L_{m, k}$ ist der Zerlegung $V_{m+1, k+1} / V_{m, k}=V_{m+1,1}$ (Nr. $2 \mathrm{~g})$ homöomorph, also retrahierbar. $L_{m, m-1}$ ist der orthogonalen Gruppe $\Gamma_{m}$ (Nr. 8c) homöomorph.

44) s. ${ }^{23}$ ), Satz II.

45) s. ${ }^{23}$ ), S. 304 (Nr. 3.8).

46) vgl. Stiefel $\left.{ }^{14}\right)$, \$ 4.1. 
c) Aus Satz 11 (Nr. 10a) folgt:

Satz 18: Es gibt dann und nur dann ein $k$-Feld auf $S^{m}$, wenn $\psi_{m-1}\left(L_{m, k} / V_{m, k}\right)=0$ ist $(0<k<m)$.

Bei geradem $m$ gibt es kein $k$-Feld auf $S^{m}$ (denn es gibt nicht einmal ein tangentiales Vektorfeld); also ist $\psi_{m-1}\left(L_{m, k} / V_{m, k}\right) \neq 0$, a fortiori also $\pi_{m-1}\left(V_{m, k}\right) \neq 0$. Oder

Korollar zu Satz 18: Für ungerades $m$ und $0<k<m$ ist

$$
\pi_{m}\left(L_{m, k}\right) \neq 0 \text {. }
$$

Darin ist der Satz von Feldbau ${ }^{47}$ ) enthalten, der aussagt, daß für ungerades $m$ immer $\pi_{m}\left(\Gamma_{m}\right) \neq 0$ ist.

Satz 19: Wenn die Sphäre $S^{m}$ parallelisierbar ist, so ist die Gruppe $\Gamma_{m}$ dem topologischen Produkt $\Gamma_{m-1} \times S^{m}$ homöomorph.

Beweis: Parallelisierbarkeit ist gleichbedeutend mit der Existenz eines $(m-1)$-Feldes auf $S^{m}$, also einer Schnittfläche der Zerlegung $\Gamma_{m} \mid \Gamma_{m-1}=S^{m}$. Die Behauptung folgt also aus Satz 16.

Da die Sphären $S^{3}$ und $S^{7}$ parallelisierbar sind ${ }^{48}$ ), ist $\Gamma_{3}$ dem topologischen Produkt $\Gamma_{2} \times S^{3}$ und $\Gamma_{7}$ dem topologischen Produkt $\Gamma_{6} \times S^{7}$ homöomorph.

d) Nach Satz G, II gilt für die $n$-te Homotopiegruppe von $\Gamma_{3}$ und $\Gamma_{7}$ :

$$
\pi_{n}\left(\Gamma_{m}\right) \approx \pi_{n}\left(S^{m}\right)+\pi_{n}\left(\Gamma_{m-1}\right), \quad m=3,7 .
$$

Insbesondere ist $\pi_{2}\left(\Gamma_{3}\right) \approx \pi_{2}\left(S^{3}\right)+\pi_{2}\left(\Gamma_{2}\right) ;$ da $\Gamma_{2}$ zum 3-dimensionalen projektiven Raum $P^{3}$ homöomorph ist, folgt nach Nr. 8a $\pi_{2}\left(\Gamma_{2}\right) \approx$ $\pi_{2}\left(S^{3}\right)=0$, also $\pi_{2}\left(\Gamma_{3}\right)=0$. In Verbindung mit Satz 9 folgt hieraus für alle $n \geqslant 3$

$$
\pi_{2}\left(\Gamma_{n}\right)=0 \text {. }
$$

Ferner ist auch $\pi_{2}\left(\Gamma_{1}\right) \approx \pi_{2}\left(V_{2},{ }_{1}\right) \approx \pi_{2}\left(S^{1}\right)=0$. Also

Satz 20: Für alle orthogonalen Gruppen $\Gamma_{n}(n=1,2, \cdots)$ ist $\pi_{2}\left(\Gamma_{n}\right)=0$.

(Das ist übrigens in einem allgemeinen Satze von Cartan $^{49}$ ) enthalten.)

e) ,Ein $k$-Feld zu einem $(k+1)$-Feld ergänzen“, heißt: ein Vektorfeld angeben, welches mit den $k$ Vektorfeldern des $k$-Feldes zusammen ein $(k+1)$-Feld bildet.

47) J. Feldbau ${ }^{9}$ ), S. 1623.

$\left.{ }^{48}\right)$ s. ${ }^{14}$ ), S. 45.

49) E. Cartan, La topologie des groupes de Lie (act. scient. et industr. 358), S. 14. 
Satz 21: Man kann a) für $m \geqslant 3$ jedes $(m-2)$-Feld auf $S^{m}$ zu einem $(m-1)$-Feld, und b) für $m \geqslant 6$ jedes 2 -Feld auf $S^{m}$ zu einem 3 -Feld ergänzen.

Bemerkungen: zu a) Ein $(m-1)$-Feld auf $S^{m}$ kann man immer zu einem $m$-Feld ergänzen; zu b) Die Bedingung $m \geqslant 6$ erweist sich später (wegen Satz 27) als überflüssig.

Beweis von Satz 21: a) Wir betrachten die 3 Zerlegungen

$$
\begin{array}{ll}
3: & V_{m+1, m} / V_{m, m-1}=V_{m+1,1} \\
3^{\prime}: & V_{m+1, m} / V_{2,1}=V_{m+1, m-1} \\
3^{\prime \prime}: & V_{m+1, m-1} / V_{m, m-2}=V_{m+1,1}
\end{array}
$$

und bezeichnen die zugehörigen Projektionen bzw. mit $P, P^{\prime}$ und $P^{\prime \prime}$. Wenn man den Zerlegungsraum von $3^{\prime}$ mit $V_{m+1, m-1}$ und die Zerlegungsräume von 3 und $3^{\prime \prime}$ mit $V_{m+1,1}$ identifiziert, so gilt offenbar

$$
P=P^{\prime \prime} P^{\prime} \text {. }
$$

Wir nehmen an, es gebe auf der $S^{m}$ ein $(m-2)$-Feld; das bedeutet, daß es in $3^{\prime \prime}$ eine Schnittfläche gibt, d. h. eine Abbildung $j_{1}$ von $V_{m+1,1}$ in $V_{m+1, m-1}$, deren Spur $P^{\prime \prime} j_{1}$ die Identität $J$ von $V_{m+1,1}$ ist.

Für $3^{\prime}$ folgt aus der Homöomorphie von $V_{2,1} \mathrm{zu} S^{1}$, daß für $m \geqslant 3$

$$
\psi_{m-1}\left(V_{m+1, m} / V_{2,1}\right)=0
$$

ist, also nach Satz $\mathrm{E}$ (Korollar), daß jede Abbildung einer $S^{m}$ in $V_{m+1, m-1}$ bezüglich $3^{\prime}$ eine Spur ist. Es gibt also eine Abbildung $j$ von $V_{m+1,1}$ in $V_{m+1, m}$, für welches $P^{\prime} j=j_{1}$ ist, also ist

$$
P j=P^{\prime \prime} P^{\prime} j=P^{\prime \prime} j_{1}=J,
$$

d. h. $j$ ist eine Schnittfläche von 3 , deren Projektion bezüglich $3^{\prime}$ die Schnittfläche $j_{1}$ von $3^{\prime \prime}$ ergibt. Das ist aber gerade die Behauptung a), wenn man nur 3 und $3^{\prime \prime}$ durch die dazu homöomorphen Zerlegungen $L_{m, m-1} / V_{m, m-1}=S^{m}$ und $L_{m, m-2} / V_{m, m-2}=S^{m}$ ersetzt.

Der Beweis von b) verläuft ganz analog, wenn man von den Zerlegungen

$$
\begin{array}{ll}
\text { 3: } & V_{m+1,4} / V_{m, 3}=V_{m+1,1} \\
3^{\prime}: & V_{m+1,4} / V_{m-2,1}=V_{m+1,3} \\
3^{\prime \prime}: & V_{m+1,3} / V_{m, 2}=V_{m+1,1}
\end{array}
$$

ausgeht und berücksichtigt, daß für $m \geqslant 6$ 
ist, und somit auch

$$
\pi_{m-1}\left(V_{m-2,1}\right) \approx \pi_{m-1}\left(S^{m-3}\right)=0
$$

$$
\psi_{m-1}\left(V_{m+1,4} / V_{m-2,1}\right)=0
$$

\section{Linienelementräume der Sphären}

a) Wir untersuchen jetzt speziell den Raum $L_{m, 1}$ aller gerichteten Linienelemente der $S^{m}$.

Bei ungeradem $m$ gibt es auf der $S^{m}$ ein stetiges Vektorfeld (man kann leicht eines explizit angeben); d. h. die natürliche Zerlegung von $L_{m, 1}$ besitzt eine Schnittfläche, und aus Satz G (Nr. 9) folgt ${ }^{50}$ ):

Satz 22: Für alle Homotopiegruppen des Linienelementraumes $L_{m, 1}$ einer Sphäre ungerader Dimension $m$ gilt

$$
\pi_{n}\left(L_{m, 1}\right) \approx \pi_{n}\left(S^{m}\right)+\pi_{n}\left(S^{m-1}\right) .
$$

b) Die natürliche Zerlegung von $L_{m, 1}$ ist eine sphärische Zerlegung $3_{m}$, deren Elemente der $S^{m-1}$ homöomorph sind. $\mathrm{Zu} 3_{m}$ gehört also nach Nr. 10e eine charakteristische Zahl $k$. Man könnte leicht direkt einsehen, daß $k$ gleich der Euler'schen Charakteristik der $S^{m}$ sein muß. Wir ziehen es aber vor, ein besonders einfaches Schnittelement der Zerlegung $\mathbf{Z}_{m}$ anzugeben, aus welchem man entnimmt, daß bei geradem $m$ die Zahl $k=2$ ist (und bei ungeradem $m$ natürlich $k=0$ ).

c) Als Vorbereitung betrachten wir eine spezielle Abbildung $s$ einer Sphäre $\Sigma^{m}$ auf eine gleichdimensionale Sphäre $S^{m} . \Sigma^{m}$ sei im $(m+1)$ dimensionalen euklidischen Raum $R_{1}^{m_{+1}}$ mit den Koordinaten $u_{1}, \ldots$, $u_{m+1} \operatorname{durch} \sum_{i=1}^{m+1} u_{i}^{2}=1$ und $S^{m}$ in $R^{m+1}$ mit den Koordinaten $x_{1}, \ldots, x_{m+1}$ $\operatorname{durch} \sum_{i=1}^{m+1} x_{i}^{2}=1$ gegeben. Die Abbildung $s$

sei durch

$$
x=s(u) \quad x \in S^{m}, u \in \Sigma^{m}
$$

definiert $\left.^{51}\right)$.

$$
x_{i}=2 u_{i} u_{1}-\delta_{i 1}, \quad i=1,2, \ldots, m+1
$$

50) Ein analoger Satz gilt offenbar für den Linienelementraum jeder Mannigfaltigkeit $M^{n}$ mit der Charakteristik 0. Es ist bemerkenswert, daß in diesem Falle sowohl Homotopie- als auch Homologiegruppen des Linienelementraumes mit denen des topologischen Produktes $M n \times S n-1$ übereinstimmen (wegen der Homologiegruppen vgl. Gysin ${ }^{9}$ )).

$\left.{ }^{51}\right)$ Identifiziert man $R^{m+1}$ mit $R_{1}^{m+1}$, so ist $\mathrm{s}(u)$ derjenige Punkt von $\Sigma m$, den man durch Spiegelung von $\Sigma m$ an dem durch $u$ gehenden Durchmesser aus dem Punkt $(1,0, \ldots, 0)$ $\epsilon R_{1}^{m}$ erhält. (Diese bzw. eine ähnliche Abbildung wird von Hopf an verschiedenen Stellen, z. B. ${ }^{12}$ ), benützt.) 
Wir bezeichnen mit $\Sigma^{m-1}$ den Äquator $u_{1}=0$ von $\Sigma^{m}$ und mit $H^{m}$ die Halbsphäre $u_{1}>0$ von $\Sigma^{m}$, ferner mit $p$ den Punkt $x_{i}=-\delta_{i 1}$ von $S^{m}$. Dann gilt:

$$
s\left(\Sigma^{m-1}\right)=p
$$

und man sieht leicht, daß $H^{m}$ topologisch auf $S^{m}-p$ abgebildet wird (mit dem Grad + 1; die Orientierung der Sphären sowie der Halbsphären sei durch die natürliche Orientierung des einbettenden euklidischen Raumes bestimmt).

Antipodische Punkte von $\sum^{m}$ haben bei der Abbildung $s$ denselben Bildpunkt auf $S^{m}$. Da die Spiegelung von $\sum^{m}$ an ihrem Zentrum den Grad +1 oder -1 hat, je nachdem $m$ ungerade oder gerade ist, folgt hieraus, da $\beta$ der Abbildungsgrad von $s$ bei ungeradem $m$ den Wert 2 und bei geradem $m$ den Wert 0 hat.

d) Wir stellen $L_{m, 1}$ dar als Mannigfaltigkeit $V_{m+1,2}$ im euklidischen Raum $R^{m+1}$, d. h. als Menge aller Paare $(x, y)$ von orthogonalen normierten Vektoren $x$ und $y$ des $R^{m+1}$ (mit den Komponenten $x_{i}$ bzw. $\left.y_{i}, i=1, \ldots, m+1\right)$. Der natürlichen Zerlegung von $L_{m, 1}(m \geqslant 2)$ entspricht dann die Zerlegung $\mathbf{3}$

$$
V_{m+1,2} / V_{m, 1}=V_{m+1,1}
$$

deren Zerlegungsraum wir als Sphäre $S^{m}$ und deren Projektion wir durch

geben können.

$$
P(x, y)=x \in S^{m}
$$

$V^{m}$ sei die ,Vollkugel“ $H^{m}+\Sigma^{m-1} \subset \Sigma^{m}$ (Bezeichnungen wie in c). Wir definieren eine Abbildung $t$ von $V^{m}$ in $V_{m+1,2}$ durch

$$
\begin{aligned}
& x_{i}=2 u_{i} u_{1}-\delta_{i 1} \\
& y_{i}=2 u_{i} u_{2}-\delta_{i 2}
\end{aligned} \quad i=1, \ldots, m+1
$$

Man verifiziert, $\operatorname{da} B \sum_{i=1}^{m+1} x_{i} y_{i}=0$ und $\sum_{i=1}^{m+1} x_{i}^{2}=\sum_{i=1}^{m+1} y_{i}^{2}=1$ ist. Die Spur $P t$ dieser Abbildung ist nichts anderes als die oben $s$ genannte Abbildung; sie bildet also $H^{m}$ topologisch auf $S^{m}-p$ und $\Sigma^{m-1}$ auf $p$ (das ist der Punkt mit den Koordinaten $x_{i}=-\delta_{i 1}$ ) ab. $t$ ist also ein Schnittelement von 3 . 
Die Abbildung $t^{\prime}=r t$ von $\sum^{m-1}\left(n_{1}=0\right)$ auf das zu $x=p$ gehörige Element der Zerlegung 3 kann man als Abbildung

$$
y_{i}=2 u_{i} u_{2}-\delta_{i 2} \quad i=2, \ldots, m+1
$$

von $\sum^{m-1}$ auf die zu $V_{m, 1}$ homöomorphe Sphäre $S^{m-1}\left(\sum_{i=2}^{m+1} y_{i}^{2}=1\right)$ auffassen; sie hat nach den Überlegungen von c) bei geradem $m$ den Grad 2 und bei ungeradem $m$ den Grad 0 .

Satz 23: Die charakteristische Zahl $k$ der natürlichen Zerlegung des Linienelementraumes $L_{m, 1}$ einer Sphäre gerader Dimension hat den Wert 2 (und einer Sphäre ungerader Dimension den Wert 0).

Es sei noch bemerkt, daß man die Abbildung $t$ auch als tangentiales Vektorfeld auf $S^{m}-p$ deuten kann; man erhält dasselbe Feld, wenn man das Vektorfeld $(0,-1,0, \ldots, 0)$ in der Äquatorebene $x_{1}=0$ stereographisch vom Punkt $p$ aus auf die $S^{m}$ projiziert. Der Index der Singularität in $p$ ist dabei dasselbe wie die charakteristische Zahl $k$.

e) Folgerungen aus Satz 23. Aus Satz 14 (Nr. 10) und unter Anwendung des Hilfssatzes aus Nr. 12 folgt nun bei geradem $m$ für $L_{m, 1}$ und seine natürliche Zerlegung $L_{m, 1} / V_{m, 1}=S^{m}$ (oder $L_{m, 1} / S^{m-1}=S^{m}$ ) für $m \geqslant 2$ :

$$
\begin{aligned}
\psi_{m-1}\left(L_{m, 1} / S^{m-1}\right) & =T_{2} \pi_{m-1}\left(S^{m-1}\right) \approx 2\left(5 \approx \mathfrak{F}^{30}\right) \\
\psi_{m}\left(L_{m, 1} / S^{m-1}\right) & =T_{2} \pi_{m}\left(S^{m-1}\right)=0 \\
P \pi_{m}\left(L_{m, 1}\right) & =0 .
\end{aligned}
$$

Für die Homotopiegruppen der Dimension $m-1, m$ und $m+1$ erhält man hieraus:

Wegen $\pi_{m-1}\left(S^{m}\right)=0$ ist $P \pi_{m-1}\left(L_{m, 1}\right)=0$, also nach Satz E :

$$
\pi_{m-1}\left(L_{m, 1}\right) \approx \pi_{m-1}\left(S^{m-1}\right) / \psi_{m-1}\left(L_{m, 1} / S^{m-1}\right) \approx\left(\mathfrak{G}_{2}{ }^{30}\right) .
$$

Ebenso folgt aus $P \pi_{m}\left(L_{m, 1}\right)=0$ für $m \geqslant 4$ :

$$
\pi_{m}\left(L_{m, 1}\right) \approx \pi_{m}\left(S^{m-1}\right) / \psi_{m}\left(L_{m, 1} / S^{m-1}\right) \approx \pi_{m}\left(S^{m-1}\right) \approx \mathfrak{G}_{2} .
$$

Wegen $\psi_{m}\left(L_{m, 1} / S^{m-1}\right)=0$ ist ferner $P \pi_{m+1}\left(L_{m, 1}\right)=\pi_{m+1}\left(S^{m}\right)$, also

$$
\pi_{m+1}\left(L_{m, 1}\right) / \varphi_{m+1}\left(L_{m, 1} / S^{m-1}\right) \approx \pi_{m+1}\left(S^{m}\right) ;
$$

aber wegen $\pi_{m+1}\left(S^{m-1}\right)=0$ ist auch $\varphi_{m+1}\left(L_{m, 1} / S^{m-1}\right)=0$, somit

$$
\pi_{m+1}\left(L_{m, 1}\right) \approx \pi_{m+1}\left(S^{m}\right) \approx \mathfrak{G}_{2} \text {, für } m \geqslant 4 \text {. }
$$


Satz 24: Für den Linienelementraum $L_{m, 1}$ einer Sphäre gerader Dimension $m \geqslant 4$ gilt

$$
\pi_{m-1}\left(L_{m, 1}\right) \approx \pi_{m}\left(L_{m, 1}\right) \approx \pi_{m+1}\left(L_{m, 1}\right) \approx \mathfrak{G}_{2} .
$$

Die Tatsache, daß $\psi_{m}\left(L_{m, 1} / S^{m-1}\right)=0$ ist, können wir nach Satz E (Korollar) auch so formulieren (gilt auch für $m=2$ ):

Satz 24': Für den Linienelementraum $L_{m, 1}$ einer Sphäre gerader Dimension gilt: Jede Abbildung einer $S^{m+1}$ in $S^{m}$ ist bezüglich der natürlichen Zerlegung von $L_{m, 1}$ eine Spur.

(Für Sphären ungerader Dimension ist dieser Satz trivialerweise gültig, weil die Zerlegung von $L_{m, 1}$ eine Schnittfläche besitzt.)

\section{Ein Satz über 2-Felder auf Sphären. Nicht-Parallelisierbarkeit der Sphäre $\mathbf{S}^{5}$}

a) Satz 25: Wenn es auf der Sphäre $S^{m}$ ein 2-Feld gibt, so kann man jedes 1-Feld auf $S^{m}$ zu einem 2-Feld ergänzen.

Beweis: $m$ sei ungerade $\geqslant 5$; denn nur dann sagt der Satz etwas aus. Wir beweisen ihn in folgender Form: Es seien 2 tangentiale Vektorfelder auf $S^{m}$ gegeben; wenn man das eine der beiden zu einem 2-Feld ergänzen kann, dann auch das andere. 3 sei die Zerlegung

$$
L_{m, 2} / V_{m-1,1}=L_{m, 1}
$$

des Raumes $L_{m, 2}$; ihre Elemente sind die Teilmengen derjenigen an die $S^{m}$ tangentialen 2-Systeme, die im selben Punkt der $S^{m}$ angreifen und im ersten Vektor übereinstimmen; ihr Zerlegungsraum sei mit $L_{m, 1}$ identifiziert. 3 ist zur Zerlegung

homöomorph.

$$
V_{m+1,3} / V_{m-1,1}=V_{m+1,2}
$$

Die beiden gegebenen Vektorfelder können wir als Abbildungen von $S^{m}$ in $L_{m, 1}$ auffassen. Dann lautet die Behauptung: Wenn eine dieser Abbildungen bezüglich 3 eine Spur ist, dann auch die andere. $f_{1}, f_{2} \epsilon$ $\pi_{m}\left(L_{m, 1}\right)$ seien die Klassen dieser Abbildungen. Wenn wir zeigen können, daß $g=f_{1}-f_{2}$ bezüglich 3 eine Spur ist, so ist der Satz bewiesen.

In der natürlichen Zerlegung $3^{\prime}$ von $L_{m, 1}$ :

$$
L_{m, 1} / V_{m, 1}=S^{m}
$$

ist $P f_{1}=P f_{2}$ die Klasse der Identität von $S^{m}$, also 


$$
P g=P\left(f_{1}-f_{2}\right)=P f_{1}-P f_{2}=0,
$$

somit

$$
g \in \varphi_{m}\left(L_{m, 1} / V_{m, 1}\right) \text {. }
$$

$V_{m, 1} \subset L_{m, 1}$ sei ein bestimmtes Element der Zerlegung $3^{\prime}$; alle die zu Punkten von $V_{m, 1}$ gehörigen Elemente $V_{m-1,1}$ der Zerlegung 3 bilden zusammen eine Mannigfaltigkeit $V_{m, 2} \subset L_{m, 2}$, in welcher die Zerlegung 3 eine Zerlegung $3^{\prime \prime}$

$$
V_{m, 2} / V_{m-1,1}=V_{m, 1}
$$

induziert; $3^{\prime \prime}$ ist zur natürlichen Zerlegung von $L_{m-1,1}$ homöomorph.

Für die Klasse $g=f_{1}-f_{2} \epsilon \pi_{m}\left(L_{m, 1}\right)$ gilt $g \in \varphi_{m}\left(L_{m, 1} / V_{m, 1}\right)$; wir können sie also durch eine Abbildung von $S^{m}$ in $V_{m, 1}$ repräsentieren, und diese ist nach Satz $24^{\prime}$ bei ungeradem $m \geqslant 5$ in $3^{\prime \prime}$ (also auch in 3) - eine Spur. Nach dem grundlegenden Lemma (Nr. 3) ist also $g$ in 3 eine Spur, q.e.d.

b) Es sei $m$ ungerade, $m=2 k-1$. In einem mit festem Koordinatensystem versehenen euklidischen Raum $R^{2 k}$ sei

$$
\mathfrak{X}=\left(x_{1}, \ldots, x_{2 k}\right)
$$

der Ortsvektor der Sphäre $S^{m}: x^{2}=1$. Mit $\tilde{\mathfrak{x}}$ bezeichnen wir den Vektor

Durch $\tilde{x}$ ist wegen

$$
\tilde{\mathfrak{X}}=\left(x_{2},-x_{1}, \ldots, x_{2 k},-x_{2 k-1}\right) \text {. }
$$

$$
\tilde{\mathfrak{X}}=0
$$

ein tangentiales Vektorfeld auf $S^{m}$ gegeben. Die Frage, ob es auf $S^{m}$ ein 2-Feld gibt, ist nach Satz 25 äquivalent mit der Frage, ob sich dieses spezielle Vektorfeld $\tilde{\mathfrak{x}}$ zu einem 2-Feld ergänzen läßt, oder: ob man eine stetige Vektorfunktion $\mathfrak{y}$ von $\mathfrak{x}$ angeben kann, derart, daß für jeden Punkt $\mathfrak{x}$ der $S^{m}$ gilt

$$
\mathfrak{y}^{2}=1 \quad \mathfrak{x} \mathfrak{y}=\tilde{\mathfrak{x}} \mathfrak{y}=0 .
$$

(Wenn dies möglich ist, so hat man übrigens gerade ein 3-Feld auf $S^{m}$; der Vektor $\tilde{\mathfrak{y}}$ liefert nämlich das dritte Vektorfeld, weil offenbar

ist).

$$
\begin{aligned}
& \tilde{\mathfrak{y}^{2}}=1, \quad \mathfrak{y} \tilde{\mathfrak{y}}=0, \\
& \tilde{\mathfrak{y}}=-\tilde{\mathfrak{y y}}=0 \quad \text { und } \quad \tilde{\mathfrak{x y}}=\mathfrak{x} \mathfrak{y}=0
\end{aligned}
$$


Wir fassen nun den $R^{2 k}$ auf als unitären Raum in $k$ komplexen Dimensionen, $U_{k}$, ordnen also dem Ortsvektor $\mathfrak{x}$ des $R^{2 k}$ den Vektor $\mathfrak{u}$ mit den $k$ komplexen Komponenten

$$
u_{j}=x_{2 j-1}+i x_{2 j}, \quad j=1, \ldots, k
$$

zu und dem Vektor $\mathfrak{y}=\left(y_{1}, \ldots, y_{2 k}\right)$ den Vektor $\mathfrak{v}$ mit den Komponenten

$$
v_{j}=y_{2 j-1}+i y_{2 j}, \quad j=1, \ldots, k \text {. }
$$

Die Bedingungen $\mathfrak{x}^{2}=\mathfrak{y}^{2}=1$ bedeuten dann

$$
\mathfrak{u} \overline{\mathfrak{u}}=\mathfrak{v} \overline{\mathfrak{v}}=1,
$$

und die Bedingungen $\mathfrak{x y}=\tilde{\mathfrak{x}} \mathfrak{y}=0$ gehen wegen

über in

$$
\mathfrak{u} \overline{\mathfrak{v}}=\mathfrak{x} \mathfrak{y}+i \tilde{\mathfrak{x}} \mathfrak{y}
$$

$$
\mathfrak{u} \overline{\mathfrak{v}}=0 .
$$

Damit ist gezeigt:

Satz 26: Es gibt dann und nur dann ein 2-Feld auf der Sphäre $S^{2 k-1}$, wenn man im k-dimensionalen unitären Raum $U_{k}$ eine für $\mathfrak{u} \overline{\mathfrak{u}}=1$ definierte stetige Vektorfunktion $\mathfrak{v}$ des Ortsvektors $\mathfrak{u}$ finden kann, für welche $\mathfrak{v} \overline{\mathfrak{v}}=1$ und $\mathfrak{u} \overline{\mathfrak{v}}=0$ ist.

c) Im Falle $m=5(k=3)$ können wir die Frage nach einem 2-Feld auf $S^{m}$ nochmals anders formulieren:

Ganz analog zur Darstellung der orthogonalen Gruppe $\Gamma_{2}$ als Vektormannigfaltigkeit $V_{3,2}$ kann man nämlich die Gruppe $A_{2}$ aller 3-reihigen unitär-unimodularen Matrizen darstellen als Mannigfaltigkeit aller im Ursprung von $U_{3}$ angreifenden unitär-orthogonalen Vektorpaare. Die zum Wirkungsraum $S_{5}$ von $A_{2}$ (vgl. Nr. $8 \mathrm{~d}$ ) gehörige Zerlegung von $A_{2}$,

$$
A_{2} / A_{1}=S^{5}
$$

entsteht in dieser Mannigfaltigkeit, wenn man jeweils alle diejenigen Vektorpaare zu einem Element der Zerlegung zusammenfaßt, die denselben ersten Vektor haben (man vgl. die Zerlegung $V_{3,2} / V_{2,1}=V_{3,1}$, die zu $\Gamma_{2} / \Gamma_{1}=S^{2}$ homöomorph ist); bezeichnen wir diesen Vektor mit $\mathfrak{u}$, so bedeutet die in Satz 26 genannte Vektorfunktion $\mathfrak{v}$ von $\mathfrak{u}$ nichts anderes als eine Schnittfläche der Zerlegung $A_{2} / A_{1}=S^{5}$. Wir sehen somit: 
Satz $26^{\prime}$ : Falls es auf der Sphäre $\$^{5}$ ein 2-Feld gibt, so besitzt die Restklassenzerlegung $A_{2} / A_{1}=S^{5}$ der unitär-unimodularen Gruppe $A_{2}$ eine Schnittfläche (und umgekehrt).

d) Auf Grund einer Untersuchung von Pontrjagin können wir die Frage der Existenz einer solchen Schnittfläche weiter verfolgen. Pontrjagin hat nämlich gezeigt ${ }^{52}$ ): $A_{1}^{\prime}$ sei eine bestimmte Restklasse der Zerlegung $A_{2} / A_{1}=S^{5} ;$ es gibt eine wesentliche Abbildung von $S^{4}$ auf $A_{1}^{\prime}$, die in $A_{2}$ zusammenziehbar ist. (Diese Abbildung sowie ihre Zusammenziehung werden dabei explizit angegeben, und man stellt übrigens leicht fest, daß es sich bei dieser Konstruktion, wenn wir unsere Terminologie verwenden, um ein Schnittelement der Zerlegung handelt, dessen Rand die wesentliche Abbildung von $S^{4}$ auf $A_{1}^{\prime}$ ist.) Das bedeutet

$$
\psi_{4}\left(A_{2} / A_{1}\right) \neq 0
$$

die Zerlegung $A_{2} / A_{1}=S^{5}$ besitzt also keine Schnittfläche, und daraus folgt wegen Satz $26^{\prime}$ :

Satz 27: Es gibt auf der 5-dimensionalen Sphäre $S^{5}$ kein 2-Feld.

Korollare: a) Die Sphäre $S^{5}$ ist nicht parallelisierbar. $\left.{ }^{53}\right)-$

b) $\psi_{4}\left(\Gamma_{5} / \Gamma_{4}\right) \neq 0$.

e) Aus der Tatsache, daß $\psi_{4}\left(A_{2} / A_{1}\right) \neq 0$, genauer

$$
\psi_{4}\left(A_{2} / A_{1}\right)=\pi_{4}\left(A_{1}\right) \approx \pi_{4}\left(S^{3}\right) \approx \mathfrak{G}_{2}
$$

ist, folgt für die unitär-unimodularen Gruppen:

Wegen $P_{\pi_{4}}\left(A_{2}\right)=0$ ist

also nach Satz 10:

$$
\pi_{4}\left(A_{2}\right) \approx \pi_{4}\left(A_{1}\right) / \psi_{4}\left(A_{2} / A_{1}\right)=0
$$

$$
\pi_{4}\left(A_{s}\right)=0 \text { für alle } s \geqslant 2 ;
$$

$\left.{ }^{52}\right)$ L. Pontrjagin, Über die topologische Struktur der Lie'sehen Gruppen (comm. math. helv. 13, S. 277-283). Das wichtige Ergebnis, daß $A_{2}$ nicht dem topologischen Produkt $S^{3} \times S^{5}$ homöomorph ist, hat Pontrjagin ohne Beweis schon früher ausgesprochen: Homologies in compact Lie groups (Recueil math. de Moscou, Bd. 6 [48], 389-422), S. 417.

${ }^{53}$ ) Wir weisen darauf hin, daß es nicht bekannt ist, ob außer $S^{1}, S^{3}$ und $S^{7}$ (vgl. ${ }^{14}$ ), S. 45) noch andere Sphären parallelisierbar sind; hingegen hat E. Stiefel (Comm. math. helv. 13 [1941], 201-218) bewiesen, daß unter den reellen projektiven Räumen höchstens die der Dimension $n=2^{\lambda}-1$ parallelisierbar sein können. 
daraus folgt, daß keine Gruppe $A_{s}(s \geqslant 2)$ einem topologischen Produkt homöomorph sein kann, in welchem ein Faktor eine $S^{3}$ ist. (Diese Konsequenzen, die wir hier nur in unserer Terminologie formuliert haben, bilden zusammen mit der oben genannten Konstruktion den Inhalt der zitierten Arbeit ${ }^{52}$ ) von Pontrjagin.)

f) Aus $\psi_{4}\left(A_{2} / A_{1}\right) \approx \mathfrak{G}_{2}$ folgt ferner

also

$$
\pi_{5}\left(S^{5}\right) / P \pi_{5}\left(A_{2}\right) \approx \mathfrak{G}_{2}
$$

Wegen

ist

$$
P \pi_{5}\left(A_{2}\right)=2 \pi_{5}\left(S^{5}\right) \approx \mathfrak{5}
$$

$$
\pi_{5}\left(A_{1}\right) \approx \pi_{5}\left(S^{3}\right)=0
$$

also

$$
\varphi_{5}\left(A_{2} / A_{1}\right)=0
$$

$$
P \pi_{5}\left(A_{2}\right) \approx \pi_{5}\left(A_{2}\right) \approx \mathfrak{G}
$$

Die Klassen der Abbildungen $f$ von $\mathcal{S}^{5}$ in $A_{2}$ sind also charakterisiert durch eine gerade Zahl, nämlich durch den Grad der Abbildung $P f$ von $S^{5}$ auf $S^{5}$.

$\operatorname{Da} \beta \pi_{5}\left(A_{2}\right) \approx\left(5\right.$ ist, folgt übrigens auch ohne Kenntnis von $\psi_{4}\left(A_{2} / A_{1}\right)$ d. h. ohne Benützung des Satzes von Pontrjagin daraus, daß $\psi_{4}\left(A_{2} / A_{1}\right)$ höchstens eine zyklische Gruppe der Ordnung 2 sein kann.

Satz 28 (vgl. Satz 10): Für die unitär-unimodularen Gruppen $A_{s}(s \geqslant 2)$ gilt

a) $\pi_{4}\left(A_{8}\right)=0 \quad$ (Pontrjagin).

b) $\pi_{5}\left(A_{8}\right) \approx \mathfrak{G}$.

\section{Felder von Flächenelementen auf Sphären}

a) Wir bezeichnen mit $E_{m, 2}$ das Büschel aller 2-dimensionalen, orientierten Ebenen des $R^{m}$ durch einen festen Punkt 0 des $R^{m}$ (auch ,Flächenelemente" genannt, genauer: orientierte Flächenelemente); als Mannigfaltigkeit aufgefaßt, ist $E_{m, 2}$ dem Zerlegungsraum einer retrahierbaren Zerlegung von $V_{m, 2}$ homöomorph (Nr. $2 \mathrm{~h}$ ):

$$
V_{m, 2} / V_{2,1}=E_{m, 2} \text {. }
$$

(Da $V_{2,1}$ zur $S^{1}$ homöomorph ist, folgt hieraus leicht, daß für $n \geqslant 3$ $\pi_{n}\left(E_{m, 2}\right) \approx \pi_{n}\left(V_{m, 2}\right)=\pi_{n}\left(L_{m-1,1}\right)$ ist. $)$ 
b) $F_{m}$ sei die Mannigfaltigkeit aller an die $S^{m}$ tangentialen Flächenelemente; sie tritt auf als Zerlegungsraum folgender Zerlegung $3^{\prime}$ von $L_{m, 2}$ : Man fasse jeweils alle die an die $S^{m}$ tangentialen 2-Systeme zusammen, die im selben Punkt der $S^{m}$ angreifen und dort dasselbe Flächenelement aufspannen (vgl. Nr. 2i) :

$$
3^{\prime}: \quad L_{m, 2} / V_{2,1}=F_{m}
$$

Wenn eine Abbildung $F$ eines Raumes $X$ in $F_{m}$ bezüglich $3^{\prime}$ Spur von $f$ ist, so bedeutet das: für jedes $x \in X$ liegt das 2-System $f(x)$ im Flächenelement $F(x)$. Wir sagen dann, $F$ sei ,durch $f$ aufgespannt".

c) $F_{m}$ besitzt eine natürliche Zerlegung $3^{\prime \prime}$, deren Elemente die Büschel $E_{m, 2}$ aller im gleichen Punkt der $S^{m}$ angebrachten tangentialen Flächenelemente, und deren Zerlegungsraum die Sphäre $S^{m}$ ist (vgl. Nr. 2i)

$$
3^{\prime \prime}: \quad F_{m} / E_{m, 2}=S^{m}
$$

Ein Feld von Flächenelementen auf $\mathcal{S}^{m}$ bedeutet eine Schnittfläche von $3^{\prime \prime}$.

Ist $P^{\prime}$ bzw. $P^{\prime \prime}$ die zu $3^{\prime}$ bzw. $3^{\prime \prime}$ gehörige Projektion, und $P$ die Projektion in der natürlichen Zerlegung 3 von $L_{m, 2}$

so gilt

$$
\text { 3: } \quad L_{m, 2} / V_{m, 2}=S^{m}
$$

d) Wegen

$$
P=P^{\prime \prime} P^{\prime} \text {. }
$$

$$
\psi_{m-1}\left(L_{m, 2} / V_{2,1}\right)=0, \quad m \geqslant 3,
$$

ist jede Abbildung der $S^{m}$ in $F_{m}$ eine Spur bezüglich $3^{\prime}$. Wir nehmen nun an, $3^{\prime \prime}$ besitze eine Schnittfläche $j_{1}$, d. h. es gebe eine Abbildung $j_{1}$ der $S^{m}$ in $F_{m}$, derart, daß $P^{\prime \prime} j_{1}$ die Identität der $S^{m}$ sei. Dann ist $j_{1}$ Spur in $3^{\prime}$, d. h. es gibt eine Abbildung $j$ der $S^{m}$ in $L_{m, 2}$, derart, daß

also

$$
P^{\prime} j=j_{1}
$$

$$
P j=P^{\prime \prime} P^{\prime} j=P^{\prime \prime} j_{1}
$$

die Identität der $S^{m}$ ist: $j$ ist Schnittfläche von 3 . 
Damit ist gezeigt:

Satz 29: Jedes Feld von Flächenelementen $\left.{ }^{54}\right)$ auf $S^{m}$ kann von einem 2-Feld auf $S^{m}$ aufgespannt werden $(m \geqslant 3)$.

Korollare: a) Auf einer Sphäre gerader Dimension $\geqslant 4$ gibt es kein Feld von Flächenelementen.

Aus Satz 27 folgt ferner: b) Auf der Sphäre $S^{5}$ gibt es kein Feld von Flächenelementen.

Aus der letzten Aussage kann man wegen des in Nr. $2 \mathrm{f}$ beschriebenen Zusammenhanges zwischen der Sphäre $S^{5}$ und der komplexen projektiven Ebene $K_{2}=P_{2}\left(\mathfrak{S}_{2}\right)$ leicht folgern, daß es auch in der komplexen projektiven Ebene $K_{2}$ kein Feld von Flächenelementen gibt.

In diesen Ergebnissen hat man unter Flächenelementen immer orientierte Flächenelemente zu verstehen; man kann aber, wie leicht zu sehen, jedes Feld von nichtorientierten Flächenelementen auf einer Sphäre ,orientieren“ (d. h. jedem Flächenelement des Feldes eine seiner beiden Orientierungen zuordnen, so daß ein Feld von orientierten Flächenelementen entsteht). Satz 29 und seine Folgerungen gelten also unverändert auch für Felder vor nichtorientierten Flächenelementen.

s4) gemeint sind immer tangentiale Flächenelemente der $\mathbf{S} m$. 


\section{Lebenslauf}

Ich wurde am 31. März 1917 in Bern geboren. Ich habe in Bern die Primarschule, dann das Progymnasium und die Literarschule des städtischen Gymnasiums besucht und im Herbst 1935 die Maturitätsprüfung in humanistischer Richtung bestanden. In den Jahren 1935-1939 habe ich an der Abteilung für Mathematik und Physik der Eidgenössischen Technischen Hochschule studiert und dort im Herbst 1939 das Diplom als Mathematiker erworben. Seither bin ich Assistent für höhere Mathematik bei Herrn Prof. Dr. W. Saxer und beschäftige mich gleichzeitig unter der Leitung von Herrn Prof. Dr. H. Hopf mit Fragen aus dem Gebiete der Topologie.

Herrn Prof. Dr. H. Hopf verdanke ich auch die Anregung zu dieser Arbeit; ich möchte ihm an dieser Stelle für sein ständiges Interesse, sowie für viele wertvolle Ratschläge und Hinweise herzlich danken.

B. Eckmann. 